Luca Tratschin*

\title{
Das Publikum und die Ausdifferenzierung eines Verfahrenssystems. Eine verfahrenssoziologische Analyse der Schlichtung zu „Stuttgart 21“
}

https://doi.org/10.1515/sosys-2017-0009

Zusammenfassung: In dem Beitrag wird die Schlichtung zum Bahnneubauprojekt Stuttgart 21 aus einer verfahrenssoziologischen Perspektive untersucht und damit die Fruchtbarkeit der Luhmann'schen Verfahrenstheorie für nicht-klassische Verfahren ausgelotet. Die These ist, dass die Schlichtung zu Stuttgart 21 eine Legitimation durch Verfahren im Luhmann'schen Sinne bewirkte. Dabei wird festgestellt, dass eine wichtige Voraussetzung, die Luhmann in seiner Verfahrenstheorie identifiziert, nicht gegeben war: Die Schlichtung beruhte nicht auf einer rechtlich fundierten Konditionalprogrammierung, die zulässige Beiträge und Darstellungen verfahrensintern definierte. Deshalb fragt der Beitrag danach, wie dieses Defizit kompensiert werden konnte und entwickelt dazu das Argument, dass dem in den Schlichtungssitzungen kommunikativ konstruierten Publikum eine wichtige Rolle in der Ausbildung schlichtungsinterner Erwartungen und damit der Ausdifferenzierung des Verfahrenssystems aus seiner Umwelt zuzuschreiben ist. Um diese Interpretation $\mathrm{zu}$ plausibilisieren, diskutiert der Aufsatz exemplarisch Resultate aus einer qualitativen Rekonstruktion des Publikumsbezugs in den Schlichtungsprotokollen zu Stuttgart 21.

Abstract: The article examines the arbitration regarding the new railway construction project Stuttgart 21 in order to explore the fruitfulness of Luhmann's procedural theory for non-classical procedures. The author argues that the Stuttgart 21 arbitration brought about legitimation through procedure in the Luhmannian sense. He notes, however, that an important prerequisite identified by Luhmann in his procedural theory was not fulfilled: the arbitration was not based on a legally sound conditional programming that defined permissible contributions and performances within the procedure. The author therefore asks, on which foundations it was possible to fulfill the function of legitimation through procedure. He develops the argument that the construction of the audience

\footnotetext{
*Korrespondenzautor: Dr. Luca Tratschin, Universität Zürich, Center for Higher Education and Science Studies (CHESS), Andreasstrasse 15, 8050 Zürich, Schweiz, email: luca.tratschin@chess.uzh.ch
} 
played an important role in the formation of internal expectations and thus in the differentiation of the process system from its environment. In order to validate this interpretation, the article discusses exemplary results from a qualitative reconstruction of the communicatively constructed audience in the minutes of the arbitration.

\section{Einleitung}

Die Bewältigung konfliktanfälliger gesellschaftlicher Aufgaben erfolgt häufig nicht mehr im Rahmen klassischer Verfahren der modernen Gesellschaft, die sich im 19. Jahrhundert ausgebildet haben (vgl. Münte 2010, 81). So werden beispielsweise öffentliche Infrastrukturprojekte wie der Ausbau von Flughäfen (Geis 2005, 2010; Münte 2012), der Bau von Bahnlinien (Della Porta/Piazza 2007), oder stadtplanerische Projekte (Magnin 2012; vgl. auch Itten 2017, 197) regelmäßig zum Gegenstand von Konflikten, die durch alternative Verfahren wie Schlichtungen oder Mediationen bewältigt werden. Vor diesem Hintergrund drängt sich die Frage auf, inwiefern die Verfahrenssoziologie Luhmanns eine fruchtbare Perspektive auf solche „nicht-klassischen“ Verfahrensformen eröffnen kann und damit auch auf gesellschaftliche Entwicklungen zu reagieren vermag. Dies ist eine der Grundfragen, die dieses Themenheft stellt.

In diesem Beitrag gehe ich diesem Interesse exemplarisch am Fall der Schlichtung zum Bahnneubau Stuttgart 21 nach, einem der wohl umstrittensten Infrastrukturprojekte der letzten Jahre im deutschsprachigen Raum. Diese Schlichtung stellt nicht nur aufgrund ihres Bekanntheitsgrades einen interessanten Fall dar, sondern auch, weil journalistische und wissenschaftliche Kommentatoren bereits die Deutung präsentiert haben, dass die Schlichtung Legitimation im Sinne der Luhmann'schen Verfahrenstheorie geleistet habe. Während allerdings bereits angedeutet wurde, dass das Schlichtungsverfahren zu Stuttgart 21 Legitimation durch Verfahren leistete, wurde bislang nicht untersucht, auf welchen kommunikativen Voraussetzungen dies beruhte. Dieser Frage gehe ich in diesem Beitrag nach.

Nach einem kurzen Überblick über die Entwicklung des Konflikts zu Stuttgart 21 in Kapitel 2, stelle ich in Kapitel 3 in einem ersten Schritt zentrale Merkmale der Schlichtung zu Stuttgart 21 vor dem Kontrastfall des Gerichtsverfahrens vor. Dabei weise ich insbesondere darauf hin, dass der Schlichter Heiner Geissler als konkreter und anwesender Dritter und das immer wieder thematisierte Publikum als generalisierte und abwesende Figur des Dritten wichtige Merkmale der Schlichtungsgespräche darstellten (Kapitel 3.1). In einem zweiten 
Schritt diskutiere ich Deutungen, die besagen, dass das Schlichtungsverfahren die Funktion der Legitimation durch Verfahren erfüllte. Ich schließe mich dieser Interpretation im Wesentlichen an, nehme allerdings kleinere Nuancierungen vor (Kapitel 3.2). Von dieser Einschätzung ausgehend, stelle ich in einem dritten Schritt eine zentrale Differenz des Schlichtungsverfahrens zu dem strukturell in vielen Hinsichten ähnlichen Gerichtsverfahren heraus: Die Legitimationsfunktion konnte bei Stuttgart 21 ohne Rückgriff auf die für Gerichtsverfahren typische rechtliche Konditionalprogrammierung erfüllt werden. Gerade eine autonome Form der Komplexitätsreduktion durch Verfahren und damit die Ruptur mit prä-existierenden und externen Erwartungen stellt gemäß Luhmann (1983) eine zentrale Voraussetzung zur Funktionserbringung von Verfahren dar. Anders formuliert: Verfahren müssen sich als soziale Systeme aus ihrer Umwelt ausdifferenzieren können, um als solche zu funktionieren (Kapitel 3.3). Ein Merkmal dafür liegt darin, dass Verfahren eigene Erwartungen bezüglich der Angemessenheit kommunikativer Beiträge ausbilden und mit verfahrensexternen Rollenerwartungen und Relevanzstrukturen brechen. Ich argumentiere hierbei, dass das verfahrensintern konstruierte Publikum der Schlichtung dafür eine wichtige Rolle spielte. Dieses diente zur Ableitung autonomer Regeln der internen Informationsverarbeitung und stützte so die Ausdifferenzierung des Verfahrenssystems (Kapitel 3.4).

In Kapitel 4 stelle ich sodann sieben verschiedene Dimensionen der Publikumsthematisierung in der Schlichtung vor, die ich in einer qualitativen Analyse der stenographischen Protokolle der Schlichtungssitzungen rekonstruieren konnte. Davon diskutiere ich exemplarisch drei Formen der Publikumsthematisierung und die daraus ableitbaren Verhaltenserwartungen an die Streitparteien vertieft: das Publikum als Laienöffentlichkeit (Kapitel 4.1), als Wahrnehmungsinstanz (Kapitel 4.2) und als zeitlich unmittelbar erlebende Instanz (Kapitel 4.3). Es geht mir in diesem Kapitel einerseits darum, die Kontingenz der Publikumskonstruktion $\mathrm{zu}$ veranschaulichen. Andererseits will ich die vielfältigen Gesichtspunkte, mit denen Beiträge durch die Bezugnahme auf das Publikum diszipliniert wurden, beispielhaft aufzeigen. Der Artikel schließt mit weiterführenden Fragen für verfahrenssoziologische Untersuchungen (Kapitel 5).

Die am Fall der Schlichtung zu Stuttgart 21 rekonstruierte Bedeutung des Publikums verweist auf das Potential, die Verfahrenssoziologie stärker in Bezug zu jüngerer Forschung zur sozialen Konstruktion von Publika und deren kommunikativen Folgen in unterschiedlichen sozialen Systemen zu setzen (vgl. Bühler/ Werron 2014; Itschert/Tratschin 2017; Koloma Beck/Werron 2018; Schmidt 2017; Tratschin 2016; 2019). Bevor der soeben skizzierte Argumentationsgang entfaltet wird, gibt der Artikel im folgenden Abschnitt zunächst einen Überblick über die Auseinandersetzung um das Bahnbauprojekt Stuttgart 21. 


\section{Das umstrittene Bauprojekt „Stuttgart 21“: Ein kurzer Überblick}

Der auf der Straße und in den Medien ausgetragene Konflikt um das Infrastrukturprojekt Stuttgart 21, bei dem der Stuttgarter Kopfbahnhof in einen unterirdischen Durchgangsbahnhof umgewandelt werden (siehe die Beiträge in Brettschneider/ Schuster 2013) sollte, stand im Jahr 2010 im Fokus der gesamten deutschsprachigen Öffentlichkeit. Der Konflikt fand in einem Zeitraum statt, in dem auch in der Weltöffentlichkeit eine Vielzahl von Protesten beobachtet wurde. Die Stuttgarter Proteste reihten sich so gut in die mediale Beobachtung der Gesellschaft in diesem Zeitraum ein: In Nordafrika fanden die Proteste des arabischen Frühlings statt (Moghadam 2013, 393f.), in Spanien und Südamerika protestierten die Indignados (Castañeda 2012). In Nordamerika und Europa besetzten Occupy-Aktivisten bald öffentliche Plätze (Calhoun 2013, 27 f.) und auch die Pegida-Proteste ließen nicht lange auf sich warten (vgl. Vorländer et al. 2016). Auch wenn die Proteste in Stuttgart sich vor dem Hintergrund eines globalen Protestzyklus verorten lassen, ${ }^{1}$ so liegen seine sozial und geographisch näheren Wurzeln in einem seit mehreren Jahren schwelenden Konflikt um ein Infrastrukturprojekt, das in der öffentlichen Debatte als „Stuttgart 21“ bezeichnet wurde.

\subsection{Das Bauprojekt „Stuttgart 21“ und sein Widerstand}

Die Anfänge dieses Bauprojektes können bis ins Jahr 1994 zurückverfolgt werden, als die Ideenskizze zum Projekt Stuttgart 21 in einer Pressekonferenz vorgestellt wurde (Stuckenbrock 2013, 34). Große öffentliche Sichtbarkeit erlangte Stuttgart 21 allerdings besonders mit der Aufnahme von Bauarbeiten und den sie begleitenden Protestaktionen im Jahr 2010. Die Kritik gegen das Bauprojekt beschränkte sich dabei nicht auf die Unannehmlichkeiten einer Großbaustelle und eines städtebaulichen Eingriffs. Neben Denkmalschutzfragen und dem Wunsch nach dem Erhalt der Bäume im Park äußerten öffentliche Sprecher auch die Befürchtung, dass die Grabungsarbeiten für die unterirdische Verlegung der Durchgangsgleise das Grundwasser und insbesondere die Mineralwasserquellen schädigen könnten (vgl. Baumgarten/Rucht 2013, 107). Albrecht Göschel (2013, $164 \mathrm{f}$.) vermutet zudem den emotionalen Kern der Auseinandersetzung in den

1 Siehe dazu zum Protestjahr 2011 am Beispiel des arabischen Frühlings und der Occupy-Proteste von 2011: Greene/Kuswa (2012). Zum Konzept des Protestzyklus siehe Tarrow (1995), vgl. auch McAdam (1995). 
symbolischen Implikationen der Ersetzung eines Kopfbahnhofes durch einen Durchgangsbahnhof, der als symbolische Abwertung von Stuttgart gelesen werden könne. Wiederum andere Kritiker sahen weniger im Bahn- oder Städtebauprojekt an sich ein Problem, sondern in der Art und Weise, wie die Bevölkerung in Entscheidungen zu diesem Projekt eingebunden - besser gesagt: nicht eingebunden - wurde. Sie kritisierten besonders das Verhältnis der Politik zu den Bürgern (vgl. Baumgarten/Rucht 2013, 108). Kurz: Das Bahnprojekt war in vielen Hinsichten umstritten.

Die Kritik am Projekt setzte schon früh ein - eigentlich nahezu gleichzeitig mit der öffentlichen Vorstellung des Projektes im Jahr 1994. Bereits im November 1995 wurde die Initiative „Leben in Stuttgart - kein Stuttgart 21“ gegründet (Rucht 2012, 342, Fn. 5). In den Folgejahren wurden mehrere Klagen gegen das Bauprojekt eingereicht und Unterschriftensammlungen organisiert. Im Anschluss an den Planungsfeststellungsbeschluss für den ersten Bauschritt im Januar 2005 gingen Klagen ein, die aber vom obersten Verwaltungsgericht des Landes abgewiesen wurden. Ebenfalls wurde ein Bürgerbegehren gegen Stuttgart 21, das durch 67.000 Unterschriften unterstützt wurde, vom Stuttgarter Gemeinderat abgelehnt. Diese Ablehnung wurde mit Verweis auf schon eingegangene Verträge begründet.

Größere Sichtbarkeit - und besonders auch Sichtbarkeit über den Stuttgarter Kontext hinaus - erlangte die Kritik an dem Bauprojekt als sich in Antizipation der Aufnahme von Bauarbeiten ab dem Spätjahr 2009 eine Protestwelle entfaltete. Diese Protestwelle setzte sich zunächst zaghaft in Gang: An der ersten Montagsdemonstration am 26. Oktober 2009 nahmen gemäß der Stuttgarter Zeitung gerade einmal vier Protestierende teil (Heffner 2012, o.S.). Dies änderte sich aber rasant im Jahr 2010 mit der tatsächlichen Aufnahme der Bauarbeiten: Nun gingen Tausende gegen Stuttgart 21 auf die Straße. Zudem griffen die Protestierenden auf ein breites „repertoire of contention“ (vgl. Tilly/Tarrow 2007, 16 f.) zurück: Neben Großdemonstrationen wurden Unterschriftensammlungen, Aktionswochen, kulturelle Veranstaltungen, Sitzblockaden, Schweigemärsche und Schwabenstreiche organisiert (Stuckenbrock 2013, 42). Die größte Eskalationsstufe erreichte der Konflikt wohl am 30. September 2010, dem so genannten „Schwarzen Donnerstag“, als bei der polizeilichen Räumung des Stuttgarter Schlossgartens durch den Einsatz von Wasserwerfern und Pfefferspray über 160 Menschen verletzt wurden (vgl. Cornelius/Dehoust/Häfner 2013, 210). 


\subsection{Festgestellte Eskalation und die Durchführung einer Schlichtung}

Angesichts der zunehmenden Eskalation des Konflikts setzte sich in der Politik die Auffassung durch, dass man nicht mehr so wie bisher weitermachen könne und es wurde die Möglichkeit einer Schlichtung zur „Versachlichung“ der Debatte diskutiert. Diese Idee stieß auf große Resonanz, so dass sich bald die Frage stellte, wer die Funktion eines Schlichters übernehmen könnte. Werner Wölfle, der Fraktionsvorsitzende der Stuttgarter Grünen, brachte Heiner Geissler ins Spiel, ein Vorschlag, der von Stefan Mappus, dem damaligen Ministerpräsidenten von Baden-Württemberg (CDU), aufgegriffen wurde. Heiner Geissler - ehemaliger Bundesminister und Generalsekretär der CDU sowie attac-Mitglied - fand dabei als potentieller Schlichter sowohl politischen Zuspruch, z. B. von der Fraktion der Grünen im baden-württembergischen Landtag, als auch mediale Unterstützung: Mehrere Journalisten äußerten sich in Artikeln äußerst wohlwollend zu diesem Vorschlag und hielten Geissler für den richtigen Mann für diese Aufgabe. Auch die Konfliktparteien zeigten sich für eine Schlichtung bereit: Politikerinnen und Politiker, die Stuttgart 21 unterstützten, als auch Vertreterinnen und Vertreter der Bahn ließen sich für die Schlichtung als „Projektbefürworter“ gewinnen. Auch von der gegnerischen Seite ließen sich Vertreter aller zentralen Gruppierungen in die Schlichtung einbeziehen. Eine Ausnahme stellte allerdings die Gruppierung der „Parkschützer“ dar, die sich nicht an der Schlichtung beteiligen wollten. Die Projektgegner waren so auch - mit Ausnahme der Parkschützer - in einem breiten Spektrum vertreten. Das Schlichtungsverfahren zu Stuttgart 21 kann gewissermaßen als Versuch gelesen werden, ein Verwaltungsverfahren, das in einen politischen Konflikt mündete, mit einer alternativen und eher ad hoc durchgeführten Verfahrensform zu retten.

Die Schlichtung zum Konflikt um das Bauprojekt wurde in kürzester Zeit durch Heiner Geissler, Lothar Frick und einer Mitarbeiterin unter „chaotischen“ Bedingungen, d. h. unter hohem Zeitdruck und geringer Mittel-Ausstattung organisiert (vgl. dazu Cornelius/Dehoust/Häfner 2013, 211 f.). Im Vorfeld einigten sich die Konfliktparteien auf die Rahmenbedingungen einer Schlichtung. Als Voraussetzung für die Durchführung einer Schlichtung wurde eine so genannte Friedenspflicht vereinbart, die insbesondere den Baustopp (mit Ausnahme bestimmter Arbeiten im Gleisvorfeld des Hauptbahnhofes) ab Beginn der Schlichtung beinhaltete. Zudem einigten sich die Konfliktparteien darauf, dass in der Schlichtung sieben Experten und je drei Mitarbeiter der beiden Konfliktseiten miteinander sprechen würden. Ebenfalls wurde vereinbart, dass die Schlichtung öffentlich sein sollte und dass das Ziel in einem „Faktencheck“ bestand (und nicht etwa in einer Kompromisssuche). Schließlich sollte 
die Schlichtung durch einen Schlichterspruch von Heiner Geissler besiegelt werden. Dieser Spruch würde keine rechtliche Verbindlichkeit aufweisen, aber auf Grundlage der Schlichtungsgespräche einen Vorschlag für das weitere Vorgehen formulieren, der dann von den Konfliktparteien auch anerkannt werden sollte.

Die Schlichtung selbst fand an acht ganztägigen Schlichtungsrunden und einem Zusatztag mit Schlussplädoyers zwischen dem 22. Oktober und dem 27. November 2010 statt. Insgesamt kamen so 51 Stunden Redezeit zustande, die live im Radio und Fernsehen (auf den Sendern Phoenix und SWR) übertragen wurden. Die gesamten Schlichtungsgespräche wurden zudem von Stenographen verschriftlicht (insgesamt 1.453 Seiten) und mit Zusatzdokumenten sowie Videomitschnitten auf einer eigens für die Schlichtung geschaffenen Webseite hochgeladen. ${ }^{2}$ Neben den nicht-öffentlichen Vorgesprächen wurde jeweils im Anschluss an die Schlichtungssitzung „Manöverkritik“ geübt, um Termine abzusprechen und Lektionen für nächste Sitzungen zu ziehen. Außerdem wurde vor der Verkündigung des Geissler'schen Schlichterspruchs noch einmal „Pendeldiplomatie“ zwischen Projektbefürwortern und Projektgegnern betrieben.

Der die Schlichtung abschließende Schlichterspruch empfahl ein Projekt „Stuttgart 21 Plus“. Dieser Schlichterspruch hielt im Wesentlichen an der Fortführung des Bauprojektes Stuttgart 21 fest, machte jedoch einige zusätzliche Vorschläge. Untere anderem wurde die Durchführung von „Stresstests“ durch Bahnbetriebssimulationen empfohlen, um allfällige Nachbesserungen und allenfalls zusätzliche Baumaßnahmen vorzunehmen. Falls sich z. B. erweisen würde, dass die geplanten acht Gleise zu wenig Kapazität sicherstellten, sollte der Tiefbahnhof um ein 9. und 10. Gleis erweitert werden. Ebenfalls empfahl der Schlichterspruch, dass die frei werdenden Grundstücke auf dem nicht mehr benötigten Gleisareal in eine Stiftung überführt werden sollten, um sie der Grundstückspekulation zu entziehen. ${ }^{3}$

2 Siehe hierzu die Webseite www.schlichtung-s21.de (zuletzt aufgerufen am 02.02.2020).

3 Für den genauen Wortlaut des Schlichterspruchs und die insgesamt zwölf Änderungs- und Verbesserungsvorschläge des Schlichters Geissler siehe: Schlichtungsverfahren zu Stuttgart 21 (2010 g, 43f.). 


\section{Schlichtung zu „Stuttgart 21“ aus einer verfahrenssoziologischen Perspektive}

\subsection{Die Schlichtung zu „Stuttgart 21“ als Form der Konfliktlösung vor zwei Typen des Dritten}

Gerichtsprozesse und alternative Verfahren der Konfliktlösung wie Schlichtung oder Mediation zielen darauf ab, einen Konflikt $\mathrm{zu}$ beenden: Die Streitpartien treten in all diesen Fällen vor eine dritte Instanz (Richter, Schlichter, Mediator), vor und mit der über die Streitangelegenheit kommuniziert wird und die diesen Konflikt z. B. mit einem Gerichtsurteil, einem Schlichterspruch oder einem Kompromissvorschlag zu beenden beansprucht. ${ }^{4}$ Hinsichtlich der Inszenierung und der Rahmenbedingungen der Schlichtung zu Stuttgart 21 sind einige Ähnlichkeiten zu Gerichtsprozessen identifizierbar. ${ }^{5}$ 1) Es traten zum einen zwei Streitparteien vor eine dritte Instanz, die den Ausgang des Verfahrens mit einem Urteilsspruch beschloss. Während Gerichtsurteile rechtskräftig werden können, so konnte der Schlichterspruch nicht diese juristische Verbindlichkeit erzeugen. Allerdings erwies sich der Geissler'sche Schlichterspruch - wie Rucht (2012, 350) hervorhebt - als faktisch verbindlich für die Konfliktparteien (siehe dazu weiter unten). 2) Der Schlichtungsvorgang vollzog sich zum anderen - wie die Gerichtsverfahren, die Luhmann (1983) beschreibt - zu wesentlichen Anteilen als Kommunikation unter Anwesenden: Die Streitparteien und der Schlichter befanden sich im selben Raum und bezogen ihre Selbstdarstellungen aufeinander. ${ }^{6}$ Es handelte sich auch hier um die Bearbeitung und Kanalisierung eines Konflikts vor einer dritten Instanz, die das Verfahren mit einer Entscheidung beenden konnte, die Bindewirkungen auf die involvierten Parteien aufwies. 3) Es handelte sich schließlich auch um eine Darstellung für Unbeteiligte, die nicht ins Verfahren eingreifen konnten, deren Erleben man aber auch - z. B. mit Bezug auf abstrakte Symbole - mitberücksichtigte. Es war also nicht nur ein „konkreter“ Dritter in der Rolle eines Richters oder Schlichters involviert, sondern ein unbekanntes und nicht direkt beobachtbares Publikum.

\footnotetext{
4 Klassisch zur Bedeutung des Dritten in einem dyadischen Konflikt: Simmel (1992, 126f., $284 \mathrm{ff}$.).

$5 \mathrm{Zu}$ Gerichtsprozessen und Legitimation durch Verfahren siehe auch die Beiträge von Heck (2017), Liebler/Zifonun (2017), Müller (2017) und Starystach (2017) in diesem Heft.

6 Für eine interaktionssoziologische Perspektive auf Verfahren siehe insbesondere Liebler/Zifonun (2017) in diesem Heft.
} 
Das Publikum wurde dabei besonders von Schlichter Geissler immer wieder adressiert und als Instanz gewürdigt, der man seine inhaltlichen Erläuterungen und Selbstdarstellungen anzupassen habe. Die Schlichtungsteilnehmenden sollten nicht nur „verständlich sprechen“, sondern sich auch allgemein „zusammenreißen“ und die Wahrnehmungsmöglichkeiten eines zuschauenden Publikums berücksichtigen (vgl. auch Sutter 2014, 300). Die Schlichtungsteilnehmenden wirkten aber an der interaktiven Publikumskonstruktion mit, indem sie die von Geissler formulierten Publikumszumutungen in der Regel akzeptierten. So kamen sie seinen Forderungen üblicherweise widerspruchslos nach oder sie entschuldigten sich explizit und brachten den Wunsch zum Ausdruck, den formulierten Erwartungen gerecht zu werden. Heiner Geissler hielt insbesondere zu Beginn jeder Schlichtungssitzung fest, dass die Bevölkerung durch die Schlichtung informiert werden müsse und gab den Sitzungen so einen „Rahmen“, in den eine starke Publikumsorientierung eingelassen war. So z. B. zu Beginn der zweiten Sitzung:

„Ich will immer daran erinnern: Wir sind dazu da, dass wir der Bevölkerung gegenüber transparent machen, was bei Stuttgart 21 geschieht, dass wir uns bemühen, die Fakten auf den Tisch zu legen, damit die Leute auch informiert sind und sich selbst ein Urteil bilden können. Das ist unsere Aufgabe, und das haben wir beim letzten Mal ganz gut angefangen“ (Schlichtungsverfahren zu Stuttgart 212010 b, 9).

$\mathrm{Zu}$ Beginn der vierten Sitzung thematisierte er zudem, dass er die Teilnehmenden immer wieder mit Verweis auf das Publikum unterbrechen würde:

\footnotetext{
„Dann möchte ich noch etwas sagen, auch weil ich dauernd angesprochen werde, dass ich Sie ab und zu unterbreche, wenn es darum geht, in einer verständlichen Sprache die Dinge darzustellen. Ich möchte auch alle Experten hier im Raume um Verständnis darum bitten, dass das notwendig ist, weil unsere Gespräche sonst das eine wichtige Ziel verfehlen, die Zuschauer und die Zuhörer miterleben zu lassen, wie diskutiert wird, wie die Argumente aussehen“" (Schlichtungsverfahren zu Stuttgart 212010 d, 3).
}

Diese publikumsbezogene Rahmung der Situation wurde interaktiv stets wieder aktualisiert, durch explizite Anrede des Publikums von Verfahrensteilnehmenden und durch Interventionen von Geissler, die die Rahmung und ihre Anforderungen in Erinnerung rufen.

Es lässt sich in diesem Zusammenhang hervorheben, dass zwei Typen von „Dritten“ eine stark strukturierende Funktion für die Schlichtungskommunikation erfüllten: Zum einen der Schlichter Heiner Geissler als vermittelnder und anwesender Dritter (Heck 2016) und zum anderen das Publikum als ,appräsentierte“ dritte Instanz (vgl. Itschert/Tratschin 2017). Besonders der anwesende und konkrete Dritte Heiner Geissler inszenierte sich dabei stark als Vertreter dieses abwesenden und generalisierten Dritten. 
Diese starke Publikumsthematisierung, insbesondere durch den Schlichter Geissler, lässt sich auf mindestens zwei Gründe zurückführen: Zum einen kann man annehmen, dass der Schlichter selbst darauf bedacht war, seine eigene „Legitimität“ durch eine Selbstdarstellung abzusichern, die ihn gewissermaßen als Vertreter des Publikums inszenierte. Die sehr affirmative und regelmäßige Thematisierung des Publikums diente zum anderen sicherlich auch als Strategie zur Akzeptanzbeschaffung des gesamten Verfahrens, das in der Form erstmalig und eher ad hoc durchgeführt wurde.

Es hat also gute Gründe gegeben, das Publikum in der Schlichtung stark zu thematisieren. Eine wichtige Folge dieser Publikumsthematisierung, so will ich im Weiteren argumentieren, lag in der Ausbildung eigener Relevanzstrukturen des Verfahrens, die die Bindung der Teilnehmenden an den Ausgang des Verfahrens vorbereitete. Während die Publikumsthematisierung auf die Akzeptanz des Schlichters und des Verfahrens in der Bevölkerung abgezielt haben mag, so erleichterte sie es ebenfalls, innerhalb des Schlichtungsverfahrens auf autonome Weise Komplexität zu reduzieren. Damit schuf das thematisierte Publikum eine wichtige Grundlage für die Legitimation durch Verfahren. Bevor ich diese These theoretisch und empirisch plausibilisiere, will ich im nächsten Anschnitt diskutieren, ob und inwiefern man davon sprechen kann, dass die Schlichtung zu Stuttgart Legitimation durch Verfahren leistete.

\subsection{Legitimation durch Verfahren in der Schlichtung zu „Stuttgart 21“}

Betrachtet man die Schlichtung zu Stuttgart 21, so kann man mit einiger Plausibilität davon sprechen, dass sie eine „Legitimation durch Verfahren“ bewirkte, wie sie Luhmann (1983) im gleichnamigen Buch entworfen hat. Dort schlägt er vor, Verfahren mit einer zur Selbstbeschreibung der Praxis inkongruenten Perspektive $\mathrm{zu}$ beleuchten $(1983,13)$. Dabei interessiert er sich entsprechend besonders für die latente Funktion von Verfahren, und nicht für ihren offiziellen Zweck. Im Besonderen untersucht er hierbei Gerichtsverfahren, Verfahren der politischen Wahl und der Gesetzgebung sowie Verwaltungsverfahren. Die Kernfunktion von Verfahren liegt für Luhmann nun nicht darin, richtige oder wahre Entscheidungen herbeizuführen, das ist gewissermaßen ihre manifeste Funktion bzw. die offizielle Begründung, wozu Verfahren gut sind: Es sollen die richtigen Urteile gefällt, die besten Politiker gewählt und die angemessenen Gesetze erlassen werden. Die eigentliche und latente Funktion von Verfahren liegt hingegen darin, Verfahrensteilnehmende an die getroffenen Entscheidungen - wie sie inhaltlich auch immer ausfallen mögen - zu binden: Wenn jemand sich darauf einlässt, an 
einem Gerichtsverfahren, Gesetzgebungsprozessen oder an einer Wahl teilzunehmen, ist es schwierig, sich von dem Gerichtsurteil, dem verabschiedeten Gesetz oder dem Ausgang der Wahl zu distanzieren. Durch die Teilnahme am Verfahren validiert man dieses performativ und bindet sich entsprechend auch an seinen Ausgang, sofern nicht nachweislich gegen die offiziellen Regeln der Verfahrensdurchführung verstoßen wurde - wenn zum Beispiel nachweisbar ist, dass der Richter aufgrund monetärer Zuwendungen bereits im Vorhinein auf der Seite der Anklage war und das Verfahren nie ergebnisoffen war oder wenn Wahlen nachweislich manipuliert wurden.

Verfahren führen nicht zwingend dazu, dass unterlegene Teilnehmende das Ergebnis tatsächlich auf der Ebene ihres Bewusstseins hinnehmen und anerkennen. Dies ist - so Luhmann - aber auch gar nicht nötig. Es reicht im Grunde, dass Verfahrensteilnehmende sich bereits durch ihre bloße Involvierung ins Verfahren sozial nicht mehr vom Ergebnis des Verfahrensentscheids distanzieren können. Wer zum Beispiel im Gerichtsprozess oder in der politischen Wahl unterliegt, kann sehr wohl frustriert sein und insgeheim davon ausgehen, dass ihm oder ihr ungerecht getan wurde. Diese Frustration wird aber durch das als regelkonform darstellbare Verfahren sozialen Anschlüssen beraubt und man bleibt einsam damit. Mitunter deshalb richtet sich das Verfahren auch nicht bloß an die Verfahrensteilnehmenden, sondern auch an ein unbeteiligtes Publikum: Über die Darstellung des Verfahrens gegenüber einem Publikum, das sich freilich selbst nicht direkt ins Verfahren einbringen kann, wird die Vermutung plausibilisiert, dass auch unbeteiligte Dritte den in einem Verfahren getroffenen Entscheid anerkennen: „Um solche Konsensvermutungen, die Verbindlichkeit des amtlichen Entscheidens betreffen, stabilisieren zu können, muss man auch die Nichtbeteiligten am Verfahren beteiligen. Sie werden zwar nicht als Sprecher in Rollen zugelassen, aber das Verfahren ist als Drama auch für sie bestimmt“ (1983, 123).

Das Schlichtungsverfahren zu Stuttgart 21 fügt sich gut ein in die analytische Perspektive, die Luhmanns Verfahrenssoziologie eröffnet. Die beiden Konfliktparteien ließen sich auf eine Serie von Gesprächen ein, in deren Verlauf „Wahrheit“ in Form eines „Faktenchecks“ geprüft werden sollte. Beide Konfliktparteien wurden in die Koproduktion von „Fakten“ involviert und eine Verfahrensgeschichte, die am Schluss in einen Schlichterspruch mündete. Da die Konfliktparteien in das Verfahren selbst eingebunden wurden, wurde es für sie im Nachhinein schwierig, Distanz zum Verfahren und Nichtakzeptanz des Entscheids öffentlich darzustellen. Eine solche Interpretation einer gelungenen „Legitimation durch Verfahren“ durch Bindung von Verfahrensverlierern an die Entscheidung und die soziale Isolation von Protest findet sich in einem Artikel von Patrick Bahners in der Frankfurter Allgemeinen Zeitung: „Da Heiner Geißler kein Got- 
tesurteil herbeiführen kann, sollte man seine ,Schlichtung` als neues Verfahren begreifen, das nötig ist, weil die vorangegangenen Verfahren keine Legitimität geschaffen und insofern keinen Schlusspunkt gesetzt haben. Kein ,Prototyp', wie Geißler hofft, sondern ein Notbehelf, der freilich Luhmanns Definition des Verfahrens erfüllt: Die Beteiligten erhalten Rollen, können die Geschichte aufarbeiten und werden in einen Prozess verstrickt, der irgendwann seine eigene Geschichte hat, so dass im Lichte der Erfahrungen im Prozess Erwartungen korrigiert werden können. Und am Ende muss eine Entscheidung stehen, für oder gegen den Neubau“ (Bahners 2010, o.S.).

Auch Dieter Rucht kommt zum Schluss, dass das Schlichtungsverfahren zu Stuttgart 21 in wesentlichen Hinsichten dem Luhmann'schen Verfahrensverständnis entsprach und eine Legitimation im Sinne der Bindung aller Teilnehmenden an den kollaborativ erwirkten Ausgang bewirkte: „Was hier als Ratschlag erscheint, hatte angesichts der Umstände des Verfahrens und der Rede von einem Schlichterspruch faktisch den Status eines Urteils, war also weder Mediation noch Schlichtung noch Verhandlung (...). Damit war genau das erreicht worden, was mit der ,Legitimation durch Verfahren“ (Luhmann 1983) beabsichtigt ist: Durch ihre Verfahrensbeteiligung waren die Projektgegner zur Hinnahme des Ergebnisses geradezu verdammt, um am Ende nicht als Querulanten, Starrköpfe, Fundamentalisten usw., als unfähig und unwillig zum politischen Kompromiss, zu erscheinen“ (Rucht 2012, 350).

Im Anschluss an das Verfahren zeigten sich die Projektbefürworter sehr zufrieden über die Durchführung und den Ausgang der Schlichtung. Dies ist nicht besonders erstaunlich, wenn man berücksichtigt, dass die Projektbefürworter ihre Position im Großen und Ganzen als bestätigt betrachten konnten. Dagegen hält Rucht es für bemerkenswerter, dass auch die - eigentlich unterlegenen - Projektgegner, die an dem Verfahren teilnahmen, die Schlichtung als Erfolg werteten und den Schlichterspruch öffentlich akzeptierten. So hielt zum Beispiel Winfried Kretschmann als Vertreter der Projektgegner fest: „Zuerst einmal muss ich betonen, dass die ganze Schlichtung aus meiner Sicht ein Erfolg war“ (Stuttgarter Nachrichten (ohne Datum), zitiert nach Rucht 2012, 346).

Allerdings äußerten sich Gegner des Projektes Stuttgart 21, die wie z. B. die Parkschützer nicht selbst am Verfahren teilnahmen, kritisch über die Schlichtung und es fanden weiterhin Protestaktionen statt, wenn auch mit wesentlich geringeren Teilnehmerzahlen als im Jahr 2010. In diesem Jahr wurden viele verschiedene Protestveranstaltungen abgehalten, die im Oktober 2010 in einer Massendemonstration ein Teilnehmermaximum von ca. 63.000 (Polizeiangaben) bis 150.000 Teilnehmenden (Aktivistenschätzung) erreichte (hil/DPAD/dpa 2010). Es fanden auch im Jahr 2018 weiterhin Proteste in der Form von Montagsdemonstrationen statt, die an der 400. Montagsdemonstration wieder etwa 1000 Protestie- 
rende mobilisierte - dreimal so viel wie in diesem Jahr üblich, wie die Stuttgarter Zeitung in ihrer Online Ausgabe am 15. Januar 2018 festhält (Baur 2018).

Vor dem Hintergrund der obigen Darstellung, können die von Bahners und Rucht präsentierten Einschätzungen zur Legitimation durch Verfahren bei Stuttgart 21 zwar aufrechterhalten werden, aber es lohnt sich, sie etwas zu qualifizieren: Die Schlichtung hat die Projektgegner sozial nicht vollständig sozial isoliert, aber erheblich und folgenreich marginalisiert: Die Projektgegner konnten nun nicht mehr damit rechnen, mit ihren unterlegenen Argumenten Gehör zu finden. Widerspruch gegen Stuttgart 21 wurde und wird zwar nach wie vor geäußert, seine Anschlussfähigkeit bei den beteiligten Protestgruppen, Parteien und Verbänden wurde jedoch durch die Schlichtung erheblich gemindert.

Die Schlichtung zu Stuttgart 21 hat nachweislich die Vertreter der Bewegung und die Parteien und Verbände, die sie repräsentierten, in den Schlichtungsgesprächen an ihren Ausgang gebunden. Ihre Anerkennung der Schlichtung und die Bereitschaft, daran teilzunehmen, hat sie der Chance beraubt, sich im Nachhinein plausibel davon $\mathrm{zu}$ distanzieren. ${ }^{7}$ Da soziale Bewegungen aber im Unterschied besonders zu formalen Organisationen - sich intern nicht gleichermaßen an Entscheidungen binden können (Tratschin 2016, 200f.), mussten Aktivisten wie die Parkschützer, die sich durch die Schlichtungsteilnehmenden nicht vertreten sehen mussten, sich nicht gleichermaßen als durch die Schlichtung gebunden betrachten. Verfahren der (alternativen) Konfliktlösung, die es mit sozialen Bewegungen anstatt mit Individuen oder Organisationen zu tun haben, können deshalb nicht gleichermaßen erwarten, dass sich die gesamte Bewegung durch die in einer Schlichtung (oder einem Gerichtsprozess) vertretenen Aktivisten festlegen lässt. Abgesehen von dieser Komplikation für den Fall sozialer Bewegungen hat aber die Schlichtung empirisch dennoch Stuttgart 21 als Konflikt maßgeblich entschärft, indem der sozialen Bewegung im Anschluss Teilnehmende, Akzeptanz und Aufmerksamkeit entzogen wurden.

Nach der Schlichtung sanken nämlich nicht bloß die zahlenmäßigen Mobilisierungserfolge drastisch, sondern die Bewegung verlor - mit den Parteien, die sich an der Schlichtung beteiligten - ihre wichtigsten Vertreter (was sich empfindlich auf das Mobilisierungspotential auswirkte). Zudem verlor der Protest

7 Insofern schließe ich mich hier nicht der kritischen Lesart von Tilmann Sutter (2014) an, die aber nicht verfahrenstheoretisch argumentiert. Dieser schätzt die Schlichtung zu Stuttgart 21 wie folgt ein: „In der Schlichtung konnte nicht entschieden werden, ein Kompromiss zwischen den Gegnern war nicht möglich“ (Sutter 2014, 300). Zwar mochten die in die Schlichtung involvierten Projektgegner von deren Ausgang privat tatsächlich enttäuscht gewesen und kein „wahrer“ Kompromiss gefunden worden sein. An die öffentliche Anerkennung des Ausgangs der Schlichtung waren sie dennoch gebunden, wie ich in diesem Beitrag zeige. 
gegen Stuttgart 21 wohl aufgrund des durch den Schlichterspruch gesetzten Schlusssteins und sinkender Mobilisierungszahlen nicht nur an Straßenöffentlichkeit, sondern auch an medialer Sichtbarkeit. Nachdem der Schlichterspruch dem Anspruch nach einen symbolischen Schlusspunkt zu der Auseinandersetzung gesetzt hatte und die involvierten Projektgegner sich zum Verfahren bekannten, zog die Medienkarawane gewissermaßen weiter (Rucht 2012, 342). Wie bereits Lipsky (1968, 1151, siehe auch: Schmitt-Beck 1990, 642) feststellte, sind Proteste, die keine öffentliche Aufmerksamkeit erhalten, geradezu inexistent. Entsprechend war Stuttgart 21 als Thema, das einen massenmedial sichtbaren und politisch als dringlich erachteten Konflikt $\mathrm{zu}$ integrieren vermochte, vorerst entschärft.

\subsection{Ausdifferenzierung als Voraussetzung für Legitimation durch Verfahren}

Es ist nun bemerkenswert, dass die Schlichtung zu Stuttgart 21 überhaupt die teilnehmenden Parteien an ihren Ausgang binden konnte, ohne dass sie auf dieselben kommunikativen Ressourcen zurückgreifen konnte, wie dies bei den „klassischen“ Verfahren Luhmanns üblich ist: Die Schlichtung verfügte über keine präexistierenden und ausformulierten Programme, an denen sich die Durchführung orientieren konnte, wie dies z. B. bei Gerichtsprozessen aber auch bei Wahlen oder dem Gesetzgebungsprozess der Fall ist. Luhmann macht in „Legitimation durch Verfahren“ (1983) den Punkt stark, dass Verfahren ihre Legitimationsfunktion besonders deshalb erfüllen können, weil sie sich anhand eigener Strukturvorgaben als Systeme aus ihrer sozialen Umwelt ausdifferenzieren können. So hält Luhmann in einem Aufsatz zur Evolution des Rechts fest: „Solche Verfahren (...) sind Sozialsysteme auf Zeit, mit einer eigenen Struktur und einer eigenen Geschichte, mit eigener Komplexität, mit Ungewissheit der Ergebnisse und mit einer gewissen Unabhängigkeit von ihrer gesellschaftlichen Umwelt - alles Systemmerkmale, die es ermöglichen, im Verfahren eine eigene Selektionsleistung zu erbringen und zu legitimieren“ (Luhmann 1981, 19, Hervorhebung LT).

Verfahren können entsprechend ihre Legitimationsverfahren erbringen, wenn sie Themen und Personen nach Maßgabe verfahrenseigener Strukturen behandeln und so eine Eigenkomplexität, die von derjenigen ihrer Umwelt verschieden ist, erzeugen können (vgl. auch Luhmann 1983, 38 ff.). In einem Gerichtsverfahren wird man zum Beispiel nicht als ganze Person behandelt, als die man sich selbst erleben mag, sondern in der Rolle des Klägers oder Angeklagten. Wer an einem Verfahren teilnimmt, muss hinnehmen, dass seine Darstellungschancen, Partizipationsmöglichkeiten und Widerspruchsformulierungen 
gemäß verfahrenseigenen Strukturen reduziert und umdefiniert werden. Auf der Ebene der Darstellung wird so ein kleinerer Ausschnitt aus dem prinzipiell möglichen Verhaltensrepertoire relevant. Welche Sachverhalte und Begebenheiten im Verfahren relevant sind und erfolgreich thematisiert werden können, hängt ebenfalls von dem ab, was durch Strukturen des Verfahrens erwartbar wird. Ein Nachbarschaftsstreit, der vor einem Richter landet, versorgt die verfeindeten Nachbarn als Kläger und als Angeklagte mit neuen Rollen und setzt sie der Notwendigkeit aus, ihre gegenseitigen Bezichtigung in eine Form zu bringen bzw. von ihren Anwälten bringen zu lassen, die rechtlich relevant sind.

Verfahren benötigen zudem Zeit und weisen nicht nur eine sachliche, sondern auch eine zeitliche Selektivität auf. Verfahren verstricken die Teilnehmenden in eine Geschichte und im Nacheinander der Beiträge und Darstellungen bilden sich verfahrensspezifische Rollen aus. Verfahren sind so zunächst ergebnisoffen, verwickeln die Teilnehmenden aber schrittweise in eine Verfahrensgeschichte nach Maßgabe verfahrenseigener Strukturen, die sich bindend auf die Selbstdarstellungen der Streitparteien auswirken. Noch ganz nahe an Goffmans (2013) Terminologie formuliert Luhmann (1983, 45): „Durch Regeln der Irrelevanz, durch Regeln der Zulassung von Personen und der Einführung von Themen, durch Übersetzungsregeln und durch Regeln zur Definition dessen, was stört oder gar das System zerstört und was zur Vermeidung dessen getan werden kann, wird das Verfahren eingegrenzt und für einige Zeit zu einer gewissen Autonomie erweckt, bis die Entscheidung fällt“. Diese von Luhmann herausgearbeitete Systemhaftigkeit von Verfahren hat für Teilnehmende den Effekt, dass sie nach der Entscheidung, am Verfahren teilzunehmen, erfahren müssen, dass ihnen Selbstdarstellungsressourcen aus anderen Lebensbereichen entzogen werden und sie ihre Meinungen, Präferenzen und Ansichten nur durch Berücksichtigung verfahrenseigener Strukturen akzeptabel zum Ausdruck bringen können. Verfahren entfalten so nicht nur hinsichtlich einer abschließenden Entscheidung, sondern bereits auf dem Weg dorthin bindende und einschränkende Effekte.

Besonders im Kontrast zu Gerichtsprozessen fällt nun auf, dass dem Stuttgarter Schlichtungsverfahren keine vergleichbare, rechtlich verankerte Konditionalprogrammierung zur Verfügung stand. Gerade dieser Form der Programmierung schreibt Luhmann jedoch ein hohes Gewicht in der Legitimationsfunktion von Verfahren zu. So argumentiert er, „dass eine Legitimation durch Verfahren nur in Verbindung mit konditionaler Programmierung des Entscheidens institutionalisiert werden kann“ $(1983,133)$. Konditionalprogramme haben typischerweise die Form des Wenn-Dann: Immer wenn ein bestimmter Sachverhalt eintritt (zum Beispiel der Feueralarm losgeht), sollen bestimmte Folgen eintreten (zum Beispiel das Gebäude evakuiert werden). Während Konditionalprogramme, zumindest in ihrer Idealform, Aktionen nahezu „automatisch“ auf der Grundlage vergan- 
gener Ereignissen auslösen, zeichnet sich die Alternative der Zweckprogrammierung durch wesentlich offenkundigere Entscheidungselastizität der Handelnden aus, da sie auf die Erreichung einer noch unbekannten Zukunft abzielen (vgl. Luhmann 1973, 263). Während bei Konditionalprogrammen alles verboten ist, was nicht durch die Programmierung erlaubt ist, ist bei Zweckprogrammierung alles erlaubt, was nicht verboten ist (vgl. Luhmann 2000, 266): Solange bestimmte Maßnahmen als geeignete Mittel erscheinen, um einen in der Zukunft zu erreichenden Zweck zu verwirklichen, darf unter ihnen gewählt werden: Will man innerhalb einer Stunde von Luzern nach Zürich reisen, kann man dies mit dem Taxi, dem Zug, einem Bus oder einem Privatflugzeug tun, je nach Mitteln, die verfügbar sind und die man für angemessen hält.

Etwas vereinfacht formuliert, schützt die Unterstellbarkeit, dass man im Verfahrensverlauf und für die Entscheidungsfindung Konditionalprogrammen gefolgt ist und allgemeine Wenn-Dann-Regeln angewendet hat, das Verfahren vor Kritik, weil sie die Entscheidungselastizität ihrer Anwendung abdunkeln: Wenn man behaupten kann, dass man bloß so reagiert hat, wie es das Konditionalprogramm gebietet, entzieht man sich als Verfahrensveranstalter gewissermaßen der Kritik, eine Entscheidung getroffen zu haben und z. B. situativ und parteiisch gehandelt zu haben. Weiterhin ist man davon entlastet, die Verantwortung für die Folgen der Regelbefolgung zu übernehmen (Luhmann 1983, 132).

Stuttgart 21 als ,ad hoc“-Veranstaltung konnte nun gerade nicht auf eine rechtlich verankerte Konditionalprogrammierung zurückgreifen und schaffte es offenbar dennoch, sich als soziales System auszudifferenzieren und die Legitimationsfunktion zu erfüllen. So stellt sich als Anschlussfrage, welches funktionale Äquivalent $\mathrm{zu}$ rechtlicher Konditionalprogrammierung in der Schlichtung Stuttgart 21 zur Verfügung stand: Wie wurde die Einschränkung der Darstellungsmöglichkeiten unter Rückgriff auf einen „entscheidungsarm“ darstellbaren Mechanismus legitimiert? Wie wurden Regeln der Irrelevanz und Übersetzungsregeln erfolgreich etabliert?

\subsection{Publikumsbezug als Mechanismus der autonomen Komplexitätsreduktion}

Die regelmäßige Disziplinierung der Schlichtungsteilnehmenden mit Verweis auf das Publikum legt die These nahe, dass der Publikumsthematisierung eine wichtige Funktion in der Etablierung verfahrensinterner Erwartungen an angemessene Redebeiträge zukam. Die regelmäßige Thematisierung des Publikums erlaubte es, verfahrenseigene Darstellungseinschränkungen ausbilden, die als wenig „entscheidungselastisch“ unterstellt werden konnten. Die in den Schlich- 
tungsgesprächen kommunikativ hergestellte Publikumsfiktion wurde so zu einem regulativen Prinzip, das die Darstellungen der Teilnehmenden von schlichtungsexternen Rollen, z. B. als Fachexperten oder als Protestierende, entkoppelte und die Ausdifferenzierung des Verfahrens als soziales System begünstigte.

Die Schlichtung insgesamt lässt sich durch den Publikumsbezug dabei grundsätzlich eher als zweckprogrammiert begreifen, denn es waren prinzipiell alle Beiträge erlaubt, denen eine angemessene Berücksichtigung des Publikums unterstellt werden konnte (siehe oben). Allerdings lässt sich beobachten, dass besonders der Schlichter Geissler von dieser Zweckorientierung des Verfahrens eine Form der Konditionalprogrammierung ableitete, die man auf die Formel bringen könnte: „Immer wenn Teilnehmende das Publikum nicht angemessen berücksichtigten, ist eine Korrektur nötig“. In dieser Konditionalprogrammierung blieb aber insbesondere die Auslösebedingung bemerkenswert undurchsichtig: Mehrheitlich deklarierte Heiner Geissler als Agent des Publikums ihr Eintreten auf der Grundlage eigener Einschätzungen, die aber mangels Widerspruchs der Teilnehmer in die „Arbeitsübereinstimmung“ der Teilnehmenden einging (vgl. Goffman 2008, 13).

Luhmann hob bereits die Bedeutung der Darstellung für Unbeteiligte in Verfahren hervor. Allerdings interessierte er sich weniger für die kommunikative Konstruktion des Publikums bzw. der Unbeteiligten in Verfahren selbst. Wahrscheinlich geprägt durch die von ihm analysierten Verfahrenstypen, zeichnet sich die Darstellung für Unbeteiligte nach Luhmann lediglich durch „Identifikation mit einigen abstrakten Symbolen, die wenig Sicherheit über die konkreten Einstellungen und Aktionsbereitschaften des anderen Menschen vermitteln“ $(1983,125)$ aus. Beim Schlichtungsverfahren zu Stuttgart wurde nun gerade nicht ein weitgehend unbekanntes Publikum unterstellt, dem man mit dem Bezug auf abstrakte Symbole Rechnung trug. Vielmehr konstruierten die Verfahrensbeteiligten in den Schlichtungssitzungen interaktiv und explizit ein spezifisches Publikum, das man bei seinen Redebeiträgen in Rechnung stellen sollte. Immer wieder wurden die Verfahrensteilnehmenden zum Beispiel dazu angehalten, „einfach“ zu formulieren, so dass das Publikum inhaltlich mitkam, es wurde auf Interessen des Publikums verwiesen oder aufgefordert, langsamer zu sprechen. Die Rollen der Streitparteien als Verfahrensteilnehmende wurde dadurch auch interaktiv umdefiniert: Da die Konfliktparteien zur Herstellung von Transparenz immer auch ein Publikum mitadressieren sollten, wurde die direkte wechselseitige Bezugnahme aufeinander, der Streit um die Richtigkeit von Aussagen regelmäßig gestört und durchbrochen. Durch den Publikumsbezug wurde so das Spektrum akzeptabler Beiträge verengt und das Verhalten der Streitparteien neu orientiert. So wurden die Möglichkeiten von Beiträgen und Selbstdarstellungen eingeschränkt und auf den Zweck des Verfahrens zugespitzt. Die starke Kopp- 
lung der Darstellenden an ihre Konflikt- und Expertenrolle wurde zugunsten der Übernahme verfahrensinterner Rollen gelockert.

\section{Die Publikumskonstruktion der Schlichtung}

Ich habe soeben eine These zur Funktion des Publikums für die Stuttgart 21Schlichtung präsentiert, die aus der großen Bedeutung, die dem Publikum in den Schlichtungssitzungen zugewiesen wurde, resultiert. Im Folgenden werde ich zur Stützung und Vertiefung dieser Interpretation einige Befunde zur Analyse der Publikumskonstruktion bei den Stuttgart 21-Schlichtungen vorstellen.

Diese Analyse beruht auf Passagen aus den stenographischen Protokollen zu Stuttgart 21. Diese Protokolle wurden in einem ersten Schritt im Hinblick auf die Frage gelesen, mit welchen Mitteln die Teilnehmenden in ihren Selbstdarstellungen und inhaltlichen Ausführungen eingeschränkt wurden, so dass die Rollen, die sie in der Schlichtung wahrnehmen konnten, nur noch locker mit externen Rollen gekoppelt waren. ${ }^{8}$ Dabei stellten sich in der Analyse Publikumsbezüge als relevante Anlässe für eine Verhaltenskorrektur der Teilnehmenden heraus. Aus diesem empirischen Befund ergibt sich die oben präsentierte Interpretation der Bedeutung des Publikums für die Schlichtung.

Um relevante Passagen $\mathrm{zu}$ identifizieren, in denen Verhaltenswartungen der Teilnehmenden thematisiert wurden, wurden die Schlichtungsprotokolle in einem ersten Schritt vollständig gesichtet. Aus diesem Analysegang konnten bereits Schlüsselworte abgeleitet werden, mit denen das Publikum thematisiert wurde. Dies waren z.B. „Bürgerinnen und Bürger“, „Bevölkerung“, „Leute“, „Leute da draußen“, „man“, „Zuschauerinnen und Zuschauer“, „Zuhörerinnen und Zuhörer“, „Zehntausende“, „Hunderttausende“. Anhand solcher Schlüsselworte wurden 285 Textstellen identifiziert, in denen das Publikum explizit thematisiert wurde. Aus diesen Textstellen wurde unter Rückgriff auf die Analysesoftware Atlas.ti in Anlehnung an das Kodierverfahren der Grounded Theory (vgl.

8 Aus forschungspragmatischen Gründen wurde entschieden, die bereits verschriftlichten Schlichtungsprotokolle als empirisches Datenmaterial zu nehmen. Die Qualität dieser Protokolle wurde stichprobenartig anhand der einsehbaren Videomittschnitte des Verfahrens geprüft und es erwies sich, dass die Protokolle die Wortlaute einzelner Redebeiträge als auch Zwischenrufe sehr adäquat wiedergeben. Zudem werden in den Protokollen auch Zusatzinformationen wie z. B. Stimmungen im Raum („Gelächter“, „Heiterkeit“) festgehalten, die eine Kontextualisierung des Gesagten erlauben. Eine Videoanalyse wäre für eine weiterführende Analyse sicherlich interessant. Für das Forschungsinteresse dieses Artikels wurden die in den Protokollen festgehaltenen Informationen und die Adäquatheit der Wiedergabe jedoch für ausreichend befunden. 
Strauss 1998) induktiv Ausprägungen des in den Schlichtungssitzungen kollektiv konstruierten Publikums und damit verbundene Handlungsaufforderungen herausgearbeitet (siehe Tabelle 1). In Anlehnung an Kellers wissenssoziologische Diskursanalyse, die an Analyseverfahren der Grounded Theory anschließt (Keller 2008, 90 ff.), und besonders in Orientierung an seinem Konzept der Phänomenstruktur (Keller 2011, 248ff.; 2008, 86 ff.) wurde die Form der Thematisierung des Publikums in den Schlichtungsgesprächen analysiert. Diskursanalytische Verfahren sind geeignet, um verschiedene Typen schriftlich fixierter Daten - aber auch anderer Datentypen - $\mathrm{zu}$ analysieren und kommen auch in der Untersuchung von Verfahren zum Einsatz (vgl. Keller 2004, 76). ${ }^{9}$ Da die Datenmenge noch relativ überschaubar war, wurden alle identifizierten Textstellen analysiert und es konnte auf Sättigung als Kriterium zum Abbruch der Analyse verzichtet werden (vgl. dazu Strauss 1998, 61). Durch iterative Kodierschritte wurden aus dem Material zum einen eine „dimensionale Erschließung“ (Strauss 1998, 41; vgl. auch Keller 2011, 251) der Publikumsthematisierung vorgenommen (siehe oberste Zeile in Tabelle 1). Zum anderen wurde anhand unterschiedlicher ,inhaltlicher Ausführungen“ (Keller 2011, 251) dieser Dimensionen verschiedene Typen von Publikumsthematisierungen identifiziert (siehe zur Orientierung die erste Spalte von Tabelle 1).

Durch diese Analyse konnten sieben verschiedene zentrale Formen der Publikumsthematisierung induktiv herausgearbeitet werden: Diese wurden 1) als politisches Publikum, 2) als Laienpublikum, 3) als Wahrnehmungsinstanz, 4) als räumlich distanziertes Publikum, 5) als zeitlich unmittelbar erlebende Instanz, 6) als quantitative Größe und 7) als rückmeldende Instanz benannt (siehe Tabelle 1 auf S. 318-319). Auch wenn diese Formen der Publikumsthematisierungen nicht immer in Reinform vorkommen, lassen sie sich im Material hinreichend gut voneinander isolieren, um sie als distinkte Thematisierungsformen des Publikums der Stuttgart 21-Schlichtung zu behandeln. Dies lässt sich besonders an den Verhaltensanforderungen, die jeweils aus dem Publikumsbezug abgeleitet werden, festmachen. An gewissen Stellen wird gefordert, für ein unmittelbar erlebendes Publikum langsamer zu sprechen, an anderen hingegen wird gefordert, zugunsten eines Laienpublikums auf Fachbegriffe $\mathrm{zu}$ verzichten. Die thematisierten Eigenschaften des Publikums übersetzen sich so in entsprechende Verhaltensaufforderungen.

Auffällig ist dabei tatsächlich, dass das Publikum regelmäßig angeführt wird, um die Äußerungs- und Darstellungsmöglichkeiten der Teilnehmenden

9 Siehe z.B. die „ethnographische Diskursanalyse“ zum Englischen Strafverfahren bei Scheffer (2010). 
zu verknappen. Je nach hervorgehobener Dimension variiert dabei die Form der Konditionierung der Verhaltensmöglichkeiten. Es werden so entlang unterschiedlicher Dimensionen Einschränkungen des Publikums thematisiert, auf die die Schlichtungsparteien Rücksicht nehmen sollen: Ein zeitlich unmittelbar erlebendes Publikum erfordert eine bestimmte Langsamkeit des Sprechens, der zugeschriebene Laienstatus des Publikums erfordert eine angemessene Wortwahl. Die Hervorhebung der Größe des Publikums geht einher mit der Erwartung, dass die Schlichtungsteilnehmenden sich angesichts der daraus ableitbaren Wichtigkeit der Veranstaltung entsprechend würdig verhalten. Dadurch wird das Verhaltensund Ausdrucksrepertoire der Streitparteien als auch der beigezogenen Experten transformiert: Die Streitparteien sollen verständigungsorientierter und kooperativer auftreten als dies ihre Rollen in der vorausgehenden Konfliktepisode nahelegten. Die Experten wurden zudem zwar in ihrem Status als Fachverständige in den „Faktencheck“ der Schlichtung einbezogen, dabei aber eines zentralen Moments dessen beraubt, was ihr Expertentum ausmacht: ihrer Expertensprache. Dies lässt sich als eine Entkopplung der Rollendarstellung von schlichtungsexternen Rollen verstehen: Prä-existierende Rollenverständnisse als politische Kontrahenten und Experten können nur Transformations- und Relevanzregeln der Schlichtung gehorchend eingebracht werden.

Die in der Schlichtung relevant gemachten Dimensionen des Publikums sind selektiv, so dass man sagen kann, dass in den Schlichtungsinteraktionen ein schlichtungsspezifisches Publikum hervorgebracht wird, das die zulässigen Darstellungen durch kontingente Einschränkung von Möglichkeiten konditioniert. Dass das Publikum an vielen Stellen zum Beispiel als politische Öffentlichkeit von Bürgerinnen und Bürgern thematisiert wird, mag an sich nicht besonders erstaunlich sein. Es fällt aber auf, dass Publikumsangehörige eigentlich nie explizit in ihrer Rolle als Bahnkunden oder Bahnbenutzer thematisiert werden. Es konnte lediglich eine interaktiv weiter folgenlose Stelle identifiziert werden, an dem stellvertretend für „Zugbenutzer“ eine Frage gestellt wurde (Schlichtungsverfahren zu Stuttgart 212010 b, 30). Im Folgenden werden exemplarisch drei der oben genannten sieben Publikumsdimensionen, die sich aus der Analyse der Schlichtungsprotokolle ergab, genauer beleuchtet. Dabei handelt es sich um die Thematisierung als Laienöffentlichkeit, als Wahrnehmungsinstanz und als zeitlich unmittelbar erlebende Instanz. 


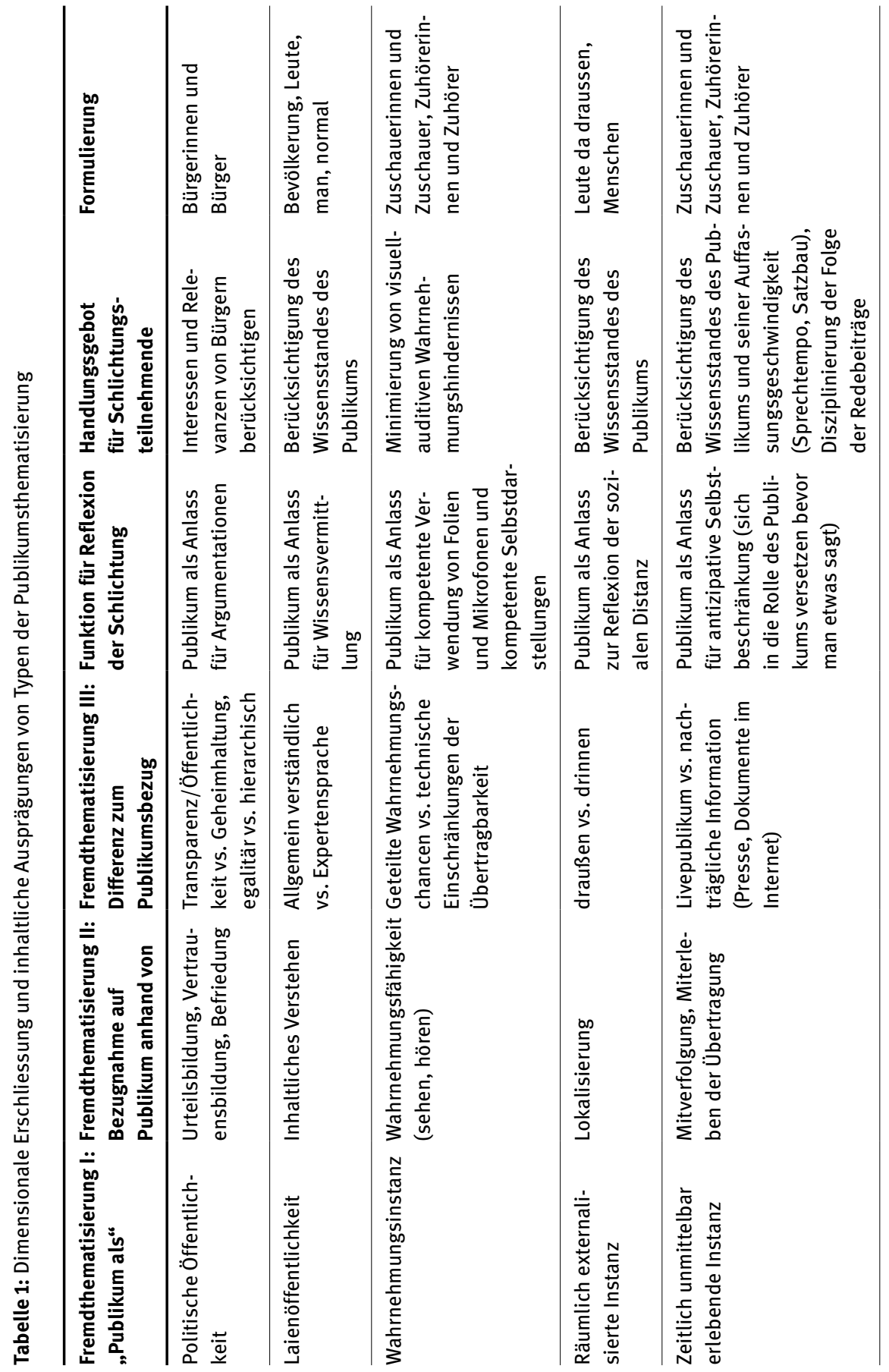




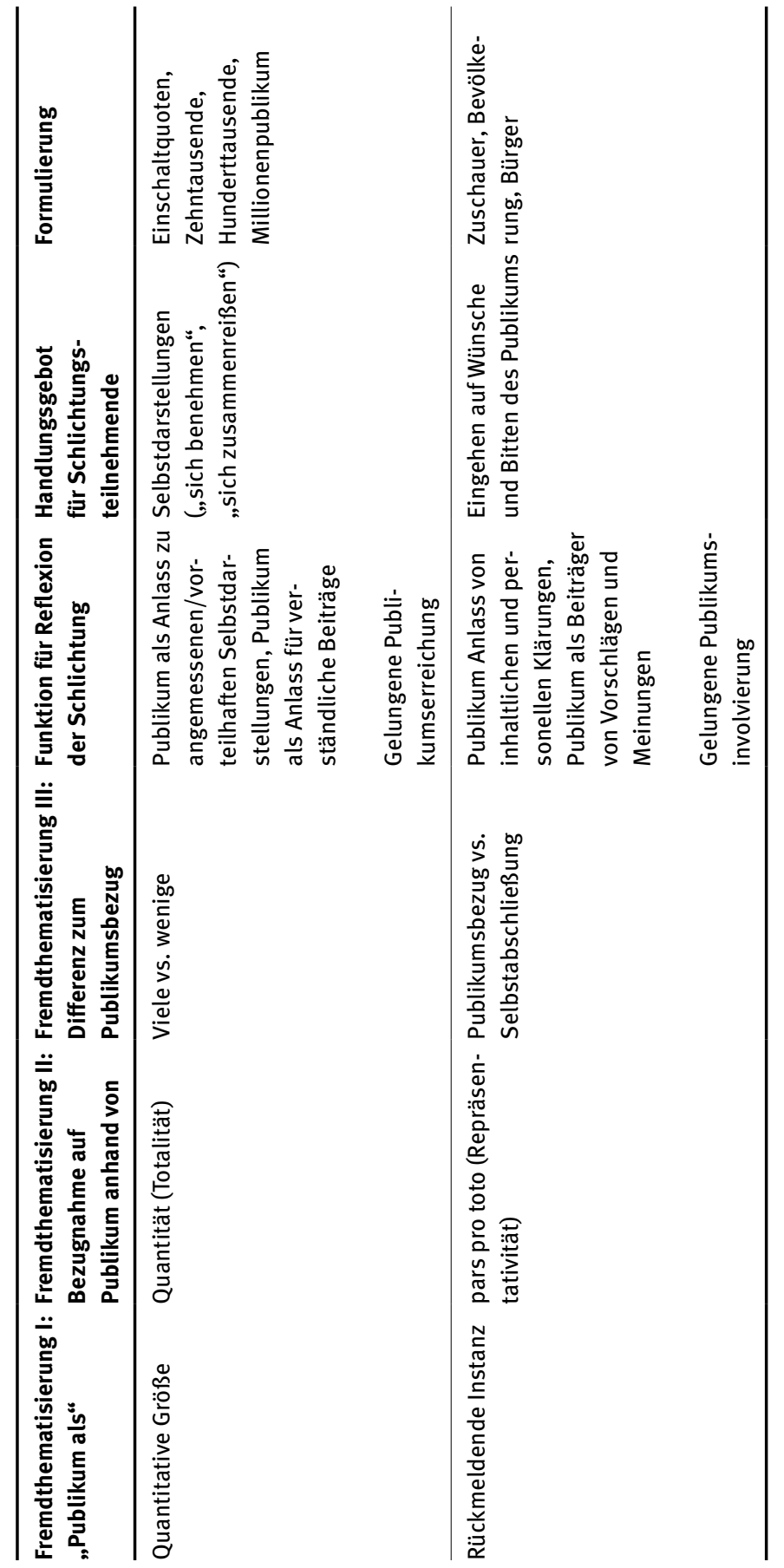




\subsection{Laienöffentlichkeit}

Wenn Schlichtungsteilnehmende das Publikum als Laienpublikum thematisieren, wird es als Adressat von Wissensvermittlung verstanden. Hierbei ist für die Schlichtungsteilnehmenden die zentrale Maxime, dass der unterstellte Wissensstand des Publikums berücksichtig werden muss. Das Publikum wird mit Ausdrücken wie „Bevölkerung“, „Leute“, „Menschen“ beschrieben. Zusätzlich wird ihnen oft das Attribut, „normal“ zu sein, zugeschrieben.

Die Hervorhebung eines Laienpublikums kontrastiert mit der Thematisierung des Publikums als politische Öffentlichkeit. Wenn das Publikum mit Ausdrücken wie „Bürgerinnen und Bürger“ als politisches Publikum thematisiert wird, stellt die Schlichtung dagegen stärker die Kompetenz der Urteilsfähigkeit des Publikums und seine Fähigkeit, Argumente selbständig zu gewichten in den Vordergrund. Sofern die Qualität des Publikums, sich aus Laien zusammenzusetzen, thematisiert wird, besteht die Verhaltenserwartung an die Schlichtungsteilnehmenden üblicherweise darin, auf Fachausdrücke und Abkürzungen zu verzichten, so dass das Publikum die Diskussion ebenfalls verstehen kann. So fordert Geissler z. B. an einer Stelle:

„Schlichter Dr. Heiner Geißler: Das ist mir schon klar. Fachausdrücke müssen hier aber allgemein verständlich erklärt werden. Wir sind hier nicht in einem Expertengremium. Hier schauen auch ein paar andere Leute zu“ (Schlichtungsverfahren zu Stuttgart 212010 a, 50, Hervorhebung LT).

Solche Forderungen werden in der Regel widerspruchsfrei akzeptiert und die Schlichtungsteilnehmenden affirmieren damit die Publikumsunterstellung im Modus der performativen Anpassung: So korrigieren sie sich in der Regel nach einer Unterbrechung, die die Berücksichtigung des Publikums anmahnt. Teilweise stellen die Teilnehmenden dar, dass sie sich wirklich bemühen wollen, verständlich zu sprechen. So z. B. Dr. Walter Wittke in der folgenden Stelle:

„Schlichter Dr. Heiner Geißler: Sie setzen Studenten voraus, die schon wissen, wie Sie reden und alles schon erahnen, was Sie möglicherweise sagen,

(Heiterkeit)

aber das ist natürlich für die Fernsehzuschauer nicht so.

Dr. Walter Wittke: Nein, ich verstehe. Das ist für mich etwas ungewohnt, Herr Dr. Geißler. Da bitte ich um Verständnis und will mich bemühen“" (Schlichtungsverfahren zu Stuttgart 21 2010 e, 13, Hervorhebung LT).

Die Verfahrensteilnehmenden akzeptieren zum einen, gemeinsam und unter informierten Streitparteien, zu einem „Faktencheck“ zu kommen. Zum an- 
deren akzeptieren sie auch, damit gleichzeitig ein Publikum von Laien zu informieren.

Sie nehmen so auch hin, dass sie einer der Sache „angemessenen“ Sprache und auch prinzipiell argumentativen Ressourcen beraubt werden: Sie können ihre Positionen und Argumente gegenüber der Gegenseite nur mit dem relativ stumpfen Mittel einer allgemein verständlichen Sprache vertreten und müssen immer auch zu einem Laienpublikum sprechen. Diese Einschränkung wird von den Teilnehmenden weitgehend akzeptiert. In der ersten Schlichtungssitzung gibt es allerdings eine kleine Streitepisode, in der Tanja Gönner kritisch auf Geisslers Unterbrechung einwendet, dass ein Faktencheck eben nicht auf eine „gewisse Fachlichkeit“ verzichten könne. Dieser Einwand wird von Geissler jedoch abgewiesen und in den folgenden Schlichtungen nicht wieder erhoben. An dieser Stelle, die Widerspruch zum Ausdruck bringt, lässt sich die Kontingenz der Publikumsthematisierung und die daraus resultierenden Einschränkungen gut demonstrieren:

\footnotetext{
„Schlichter Dr. Heiner Geißler: Darf ich noch einmal Folgendes sagen. Können Sie nicht sagen: Wir planen, dass jede halbe Stunde ein Zug von Ulm nach XY fährt, anstatt halbstündlicher Takt oder integraler Takt. Das geht nicht. Wir müssen es so machen, dass die Leute es verstehen. - Bitte, Frau Gönner.

Tanja Gönner (Projektbefürworterin): Herr Geißler, ich möchte darauf hinweisen, dass wir uns vorgenommen haben, hier Fakten zu besprechen. (...). Eine gewisse Fachlichkeit wird man in einer Fach- und Sachschlichtung, in einer Faktenschlichtung nicht vermeiden können, sondern wir werden das zulassen müssen.

Schlichter Dr. Heiner Geißler: Sie brauchen mich nicht darüber zu informieren. Es geht darum, dass wir nicht in einem Expertengremium sind, sondern Leute zuschauen und zuhören“" (Schlichtungsverfahren zu Stuttgart 212010 a, 92f., Hervorhebung LT).
}

\subsection{Publikum als Wahrnehmungsinstanz}

Das Publikum wird weiterhin regelmäßig als auditiv-visuelle Wahrnehmungsinstanz thematisiert. In dieser Hinsicht stellt sich dann die Herausforderung, so zu sprechen und sich so zu verhalten, dass das Publikum einen auch hören und sehen kann. Dazu sind besonders die richtige Verwendung von Mikrofonen und der kompetente Gebrauch von Präsentationsfolien wichtig. Die technisch bedingten audiovisuellen Wahrnehmungseinschränkungen des Publikums werden regelmäßig Anlass, um dessen Telepräsenz interaktiv zu aktualisieren und Verhaltensanpassungen einzufordern. Ein dominanter Problembezug stellt dabei die Minimierung auditiver und visueller Wahrnehmungshindernisse dar. So wird die technische Infrastruktur dazu verwendet, die Verhaltensweisen der Teilnehmen- 
den zu disziplinieren. Wer spricht, soll zum Beispiel direkt ins Mikrofon sprechen und dieses auch kompetent bedienen, d. h. vor dem Sprechen auf einen Schalter drücken, damit das Gesagte hörbar ist.

„[Geissler:] Darüber hinaus ist es wichtig, dass Sie direkt ins Mikrofon sprechen. Außerdem ist es hilfreich, wenn nicht zwei Leute oder sogar noch mehr Leute gleichzeitig sprechen. Wenn man im Chor redet, kann man nichts mehr verstehen. Für die vielen Leute, die uns zuschauen und zuhören, wäre es dann schwer, uns zu folgen“" (Schlichtungsverfahren zu Stuttgart 212010 a, 5, Hervorhebung LT).

Aber nicht nur hinsichtlich der Hörbarkeit der Beiträge muss man die Wahrnehmungsmöglichkeiten des Publikums in Rechnung stellen. Die Teilnehmenden werden regelmäßig dazu angehalten, einzuberechnen, was das Publikum sieht und dies in Einklang zu bringen mit dem, was gesagt wird. Sprechen und Sehen sollen in Übereinstimmung gebracht werden.

„Schlichter Dr. Heiner Geißler: Können Sie vielleicht mit einem Stift oder einem Pfeil zeigen, wovon Sie reden? Die Leute am Bildschirm können Ihrer sachkundigen, aber relativ zügigen Sprechweise nicht ohne Weiteres folgen, wenn sie nicht wissen, wovon Sie reden“ (Schlichtungsverfahren zu Stuttgart 21 2010, 45, Hervorhebung LT).

Zuweilen wird aber auch darauf verwiesen, dass sich das Publikum aufgrund dessen, was es sieht, Urteile über die Schlichtungsteilnehmenden fällt. Man solle so auch auf der Ebene von Körperbewegungen und -tätigkeiten das Publikum berücksichtigen. So korrigiert z.B. Geissler Tanja Gönner nach einem Wortbeitrag:

„Schlichter Dr. Heiner Geißler [zu Tanja Gönner]: Ich bedanke mich sehr. Sie hätten aber ins Publikum sehen sollen“ (Schlichtungsverfahren zu Stuttgart 212010 f, 158, Hervorhebung LT).

Ebenfalls verweist der Schlichter darauf, dass man zwar im Schlichtungssaal während der Sitzung essen könne, man aber bedenken müsse, wie Zuschauer am Fernsehen dieses „Nebenengagement“ bewerten könnten:

„[Geissler:] Wir tagen bis 14 Uhr ohne Mittagspause. Ich nehme an, dass, wenn jemand Hunger hat, er sich hinterher noch verköstigen kann. Von mir aus können Sie auch hier im Saal essen, wenn Sie glauben, dass es für Ihr Image im Fernsehen zuträglich ist“ (Schlichtungsverfahren zu Stuttgart 212010 e, 2, Hervorhebung LT).

An solchen Stellen wird das Publikum gerade in seinen Einschränkungen aber auch der prinzipiellen Fähigkeit, das interaktive Geschehen im Schlichtungssaal 
auditiv und visuell wahrnehmen zu können, relevant gemacht. Es geht hier nicht um das Bezugsproblem der Wissensvermittlung an ein Laienpublikum, sondern um das Problem der angemessenen Berücksichtigung eines - unter Einschränkungen - Sinneseindrücke empfangenden Publikums.

\subsection{Publikum als zeitlich unmittelbar erlebende Instanz}

Das Publikum stellt weiterhin durch sein synchrones Erleben der Schlichtungsvorgänge eine Herausforderung für die Darstellungen der Schlichtungsteilnehmenden dar. Dieses ist gewissermaßen der Eigenzeit der Schlichtung ausgesetzt - es ist dem Sprechtempo und der mehr oder weniger übersichtlichen Abfolge von Redebeiträgen geradezu ausgeliefert. Deshalb muss das Tempo der Schlichtung an die Verarbeitungskapazitäten des Publikums angepasst werden. In der Schlichtung wird zwar auch thematisiert, dass man über die Zeitungen oder Lektüre der Schlichtungsprotokolle nachträglich und - im Fall von Zeitungen - durch journalistische Deutungshilfen informiert werden kann. Aber in der Schlichtung wird ein Publikum von synchronen Zuschauern und Zuhörern unterstellt. Es wird so auch regelmäßig thematisiert - üblicherweise in den einleitenden Rahmungen der Schlichtungssitzungen -, dass die Schlichtung live im Fernsehen und im Radio übertragen wird. Die Schlichtungsteilnehmenden werden dabei immer wieder daran erinnert, in ihren Redebeiträgen ein synchron erlebendes Publikum mitzuberücksichtigen. Sie können bzw. sollen in ihren Darstellungen deshalb nicht auf nachträgliche und vermittelnde Deutungshilfen bauen. Die Berücksichtigung eines synchron erlebenden Publikums stellt auch eine gemeinsame Leistung der Verfahrensbeteiligten dar: Redezüge sollen „sauber“ und verständlich aneinander anschließen. Zudem ist die Konsistenz der aufeinander folgenden Beiträge ein Gebot - im Namen des Publikums:

„Schlichter Dr. Heiner Geißler: Ich versuche seit ungefähr einer halben Stunde, deutlich zu machen, dass es wirklich um verkehrliche Leistungsfähigkeit Kopfbahnhof oder Durchgangsbahnhof geht. Die Leute, die die letzten anderthalb Stunden zugehört haben, worüber wir reden, werden ja total verwirrt“ (Schlichtungsverfahren zu Stuttgart 212010 a, 88, Hervorhebung LT).

Weiterhin wird auch das Sprechtempo einzelner Redebeiträge im Namen des Publikums kritisiert und es werden Anpassungen eingefordert:

„Schlichter Dr. Heiner Geißler: Vielleicht kann man ein bisschen langsamer reden. Das ist zu schnell für denjenigen, der nicht in der Materie ist. Die Leute wollen das ja auch verstehen“ (Schlichtungsverfahren zu Stuttgart 212010 b, 47, Hervorhebung LT). 
In diesem Sinne wird auch eine zeitliche Dekompression von Fachausdrücken gefordert: Fachausdrücke sollen in ein Nacheinander von Subjekt und Prädikat übersetzt werden (damit man „mitverfolgen“ - also zeitlich nachkommen kann):

„Schlichter Dr. Heiner Geißler: Herr Dr. Bitzer, ich unterbreche Sie ungern. Aber Sie sind da besonders verdächtig, wie wir aus den früheren Diskussionen wissen. Bitte unternehmen Sie den Versuch, das, was Sie sagen, so zu formulieren, gerade komplizierte Substantive in Sätze mit Subjekt und Prädikat aufzulösen. Dann können die Leute das draußen am Bildschirm besser mitverfolgen“ (Schlichtungsverfahren zu Stuttgart 212010 c, 14, Hervorhebung LT).

Diese Dominanz eines als synchron unterstellten Publikums ist durchaus bemerkenswert, insofern ebensogut ein Publikum hätte thematisiert werden können, das sich nachträglich über die Schlichtung informiert - in Form von Zeitungslektüre oder Nachrichtensendungen, die zwar Videomittschnitte zeigen, diese aber kommentieren. Zwar waren die Einschaltquoten der Direktübertragung der Stuttgart 21 Schlichtung beim Fernsehsender Phoenix erstaunlich hoch: etwa 100.000 Zuschauer verfolgten die Übertragung (vgl. hip 2010). Im Unterschied dazu lag die Auflage der Stuttgarter Nachrichten selbst bei etwa 143.000 Exemplaren (VSZV Jahresbericht 2010, 62) und die Tagesschau auf ARD, die ebenfalls über Stuttgart 21 berichtete, erreicht ein Millionenpublikum (vgl. Das Erste 2018; Grzeschik 2018). Die Thematisierung eines synchron erlebenden Publikums und die damit einhergehenden Relevanzregeln waren somit nicht alternativlos - ebenso wie die Unterstellung eines kognitiv defizitären Laienpublikums und eines audiovisuell wahrnehmenden Publikums.

\section{Schluss}

Dieser Artikel ging davon aus, dass die Schlichtung zu Stuttgart 21 Legitimation durch Verfahren im Luhmann'schen Sinne erreichte. Das Publikum stellte gemäß der hier vertretenen Interpretation einen Mechanismus der Schlichtung dar, um eine eigenständige, von den unmittelbaren Relevanzen der Streitparteien entkoppelte Erwartungsstruktur bezüglich der Selektion und Form der Thematisierung von Beiträgen und Selbstdarstellungen aufzubauen, die nur schwer kritisierbar war: Wer wollte sich dagegen aussprechen, dass „die Leute da draußen“ einen nachvollziehbaren Einblick in die Fakten und Argumente zum politischen Konflikt um den Bau des Durchgangsbahnhofes in Stuttgart erhielten? Die Publikumsthematisierung wurde anhand dreier Formen der Publikumsthemati- 
sierung und der damit einhergehenden Verhaltenserwartungen exemplarisch diskutiert.

Der Artikel kann den Schluss ziehen, dass die analytische Perspektive, die durch Luhmanns „Legitimation durch Verfahren“ aufgemacht wird, fruchtbar auf den Fall der Schlichtung zu Stuttgart 21 angelegt werden konnte. Dies nicht unbedingt in dem Sinne, dass alternative Formen der Konfliktlösung wie die hier untersuchte, sich einfach reibungslos unter die Luhmann'sche Verfahrenssoziologie subsumieren ließen. Vielmehr erwiesen sich Kernannahmen der Verfahrenssoziologie - wie die Selbstbindung der Teilnehmenden an den Ausgang des Verfahrens und die von Luhmann herausgestellte Bedeutung rechtförmiger Programmierung - als fruchtbar, um die Funktionsweise der Schlichtung zu Stuttgart 21 weiter aufzuschlüsseln: In diesem Aufsatz wurde gezeigt, dass die Schlichtung unter Heiner Geissler Legitimation durch Verfahren im Luhmannschen Sinn bewirkt hatte. Sie fällt entsprechend in den Zuständigkeitsbereich der Verfahrenssoziologie. Ausgehend von dieser Deutung drängte sich aber dann die Frage auf, wie diese Leistung erbracht werden konnte. In diesem Punkt ging die Analyse über die bestehenden Argumente der Luhmann'schen Verfahrenssoziologie hinaus.

Ausgehend von der Abweichung des hier diskutierten Falles können Rückfragen an die Verfahrenstheorie gewonnen werden, die Chancen und Grenzen der Generalisierung dieser Theorie auf alternative Verfahren der Konfliktlösung betreffen. Bei der Schlichtung zu Stuttgart 21 wurde in diesem Beitrag besonders eine Abweichung zu den Verfahren herausgestellt, die Luhmann diskutiert: Schlichtungen wie jene zu Stuttgart 21 sind nicht rechtlich geordnet wie dies für die Verfahren gilt, die Luhmann bespricht. Es gab keine Rechtstexte, auf die in der Schlichtung verwiesen werden und anhand derer die Schlichtung konditional programmiert werden konnte. In den obigen Ausführungen wurde das Argument entwickelt, dass der interaktiv hergestellte Publikumsbezug als ein funktionales Äquivalent zur rechtlichen Ordnung des Verfahrens verstanden werden kann: Es erlaubte dem Verfahren eine eigenständige, von den Relevanzen der Streitparteien abgekoppelte Form der internen Komplexitätsreduktion. Weiterführend könnte man die Frage stellen, ob sich die Relevanz dieser Publikumsorientierung auch in anderen Fällen alternativer Konfliktregelung identifizieren lässt. Besonders interessant wäre hierbei die Frage, ob die Funktion der Legitimation durch Verfahren in Formen der Konfliktlösung, die nicht rechtlich geordnet sind und einen starken Publikumsbezug aufweisen, auf robuste Weise erfüllt werden kann, oder ob sie gegenüber den Verfahren, die Luhmann diskutiert, empirisch nachweisbare Akzeptanzprobleme hervorbringen. Eine Problemquelle könnte dabei darin liegen, dass die kollektive Publikumskonstruktion - im Unterschied zum hier präsentierten Fall - gar nicht erst gelingt. Eine andere Problemquelle 
könnte darin liegen, dass selbst gelungene Publikumskonstruktionen gegenüber der rechtlichen Konditionierung von Verfahren nicht mit derselben Zuverlässigkeit Komplexität zu reduzieren und Verbindlichkeit zu erzeugen vermögen.

\section{Literatur}

Baumgarten, Britta/Rucht, Dieter (2013): Die Protestierenden gegen „Stuttgart 21“ einzigartig oder typisch?, in: Frank Brettschneider/Wolfgang Schuster (Hrsg.), Stuttgart 21. Ein Großprojekt zwischen Protest und Akzeptanz. Wiesbaden: Springer, 97-126.

Brettschneider, Frank/Schuster, Wolfgang (Hrsg.) (2013): Stuttgart 21. Ein Großprojekt zwischen Protest und Akzeptanz. Wiesbaden: Springer.

Bühler, Martin/Werron, Tobias (2014): Zur sozialen Konstruktion globaler Märkte. Ein kommunikationstheoretisches Modell, in: Andreas Langenohl/Dietmar J. Wetzel (Hrsg.), Finanzmarktpublika: Moralität, Krisen und Teilhabe in der ökonomischen Moderne. Wiesbaden: Springer, 271-299.

Calhoun, Craig (2013): Occupy Wall Street in Perspective. The British Journal of Sociology 64, 26-38.

Castañeda, Ernesto (2012): The Indignados of Spain: A Precedent to Occupy Wall Street. Social Movement Studies 11, 309-319.

Cornelius, Sabrina/Dehoust, Eva/Häfner, Carolin (2013): „Einbahnstraßenkommunikation ist nicht die Lösung“. Fragen an Lothar Frick, in: Frank Brettschneider/Wolfgang Schuster (Hrsg.), Stuttgart 21. Ein Großprojekt zwischen Protest und Akzeptanz. Wiesbaden: Springer, 209-218.

Della Porta, Donatella/Piazza, Gianni (2007): Local Contention, Global Framing: The Protest Campaigns against the TAV in Val di Susa and the Bridges on the Messina Straits. Environmental Politics 16, 864-882.

Geis, Anna (2005): Regieren mit Mediation: Das Beteiligungsverfahren zur zukünftigen Entwicklung des Frankfurter Flughafens. Wiesbaden: VS.

Geis, Anna (2010): Beteiligungsverfahren zwischen Politikberatung und Konfliktregelung: Die Frankfurter Flughafen-Mediation, in: Peter H. Feindt/Thomas Saretzki (Hrsg.), Umwelt- und Technikkonflikte. Wiesbaden: VS, 259-274.

Goffman, Erving (2008) [1959]: Wir alle spielen Theater. Die Selbstdarstellung im Alltag. München, Zürich: Piper.

Goffman, Erving (2013) [1961]: Fun in Games, in: ders., Encounters. Two Studies in the Sociology of Interaction. Mansfield Centre: Martino Publishing, 17-84.

Göschel, Albrecht (2013): „Stuttgart 21“: Ein postmoderner Kulturkonflikt, in: Frank Brettschneider/Wolfgang Schuster (Hrsg.), Stuttgart 21. Ein Großprojekt zwischen Protest und Akzeptanz. Wiesbaden: Springer, 149-172.

Greene, Ronald Walter / Kuswa, Kevin Douglas (2012): „From the Arab Spring to Athens, From Occupy Wall Street to Moscow“: Regional Accents and the Rhetorical Cartography of Power. Rhetoric Society Quarterly 42, 271-288.

Heck, Justus (2016): Der beteiligte Unbeteiligte. Wie vermittelnde Dritte Konflikte transformieren. Zeitschrift für Rechtssoziologie 36, 58-87. 
Heck, Justus (2017): Vermittlung im Zivilverfahren. Zu Funktionen und Folgen undifferenzierter Konfliktbearbeitung, in diesem Heft.

Itschert, Adrian/Tratschin, Luca (2017): Dissent without Disaster: Konditionierte Konflikte und öffentlich appräsentierte Dritte, in: Henrik Gummert/Jelena Henkel-Otto/Dirk H. Medebach (Hrsg.), Medien und Kulturen des Konflikts. Pluralität und Dynamik von Generationen, Gewalt und Politik. Wiesbaden: Springer, 13-43.

Itten, Anatol (2017): Context and Content toward Consensus in Public Mediation. Negotiation Journal 33, 185-211.

Keller, Reiner (2004): Diskursforschung. Eine Einführung für SozialwissenschaftlerInnen, Wiesbaden: VS.

Keller, Reiner (2008): Diskurse und Dispositive analysieren. Die wissenssoziologische Diskursanalyse als Beitrag zu einer wissensanalytischen Profilierung der Diskursforschung. Historical Social Research 33, 73-107.

Keller, Reiner (2011): Wissenssoziologische Diskursanalyse. Grundlegung eines Forschungsprogramms. Wiesbaden: VS.

Koloma Beck, Teresa/Werron, Tobias (2018): Violent Conflicition. Armed Conflicts and Global Competition for Attention and Legitimacy. International Journal of Politics, Culture, and Society 31, 275-296.

Liebler, Carina/Zifonun, Dariuš (2017): Interaktionsordnung des Gerichtsverfahrens. Strukturelemente ihres Vollzugs und die Bewältigung von Identitätsproblemen, in diesem Heft.

Lipsky, Michael (1968): Protest as a Political Resource. The American Political Science Review 62, 1144-1158.

Luhmann, Niklas (1973): Zweckbegriff und Systemrationalität. Über die Funktion von Zwecken in sozialen Systemen. Frankfurt a.M.: Suhrkamp.

Luhmann, Niklas (1981): Evolution des Rechts, in: Ders., Ausdifferenzierung des Rechts. Beiträge zur Rechtssoziologie und Rechtstheorie. Frankfurt a.M.: Suhrkamp, 11-34.

Luhmann, Niklas (1983): Legitimation durch Verfahren. Frankfurt a.M.: Suhrkamp.

Luhmann (2000): Organisation und Entscheidung. Opladen: Westdeutscher Verlag.

Magnin, Chantal (2012): Zur Moderation von Nutzungsbedürfnissen: Normative Vorstellungen zur Partizipation von Bürgern bei der Stadtplanung, in: Alfons Bora/Peter Münte (Hrsg.), Mikrostrukturen der Governance. Beiträge zur materialen Rekonstruktion von Erscheinungsformen neuer Staatlichkeit. Baden-Baden: Nomos, 217-260.

McAdam, Doug (1995): „Initiator“ and „Spin-off“ Movements: Diffusion Processes in Protest Cycles, in: Mark Traugott (Hrsg.), Repertoires and Cycles of Collective Action. Durham / London: Duke University Press, 217-239.

Moghadam, Valentine M. (2013), What is Democracy? Promises and Perils of the Arab Spring. Current Sociology 61, 393-408.

Müller, Marion (2017): Keine Legitimation durch Familiengerichtsverfahren? Akzeptanzprobleme gerichtlicher Entscheidungen und Konstruktion geschlechtsdifferenter Elternschaft, in diesem Heft.

Münte, Peter (2010): Zur Rolle partizipativer Verfahren im Wandel von Politik: Rekonstruktion einer berufsbiographischen Schlüsselepisode eines Umweltmediators. Zeitschrift für Rechtssoziologie 31, 81-102.

Münte, Peter (2012): Das Mediationsverfahren als sozialtechnologische Form herrschaftstechnischer Versachlichung und inszenierter Herrschaftsfreiheit: Eine Analyse eines Entwurfs der Vereinbarung über eine Mediation zum Ausbau des Flughafens Wien, in: Alfons Bora/Peter Münte (Hrsg.), Mikrostrukturen der Governance. Beiträge zur materialen 
Rekonstruktion von Erscheinungsformen neuer Staatlichkeit. Baden-Baden: Nomos, 217-260.

Rucht, Dieter (2012): Akzeptanzbeschaffung als Legitimationsersatz: Der Fall Stuttgart 21, in: Anna Geis/Frank Nullmeier/Christopher Daase (Hrsg.), Der Aufstieg der Legitimitätspolitik. Rechtfertigung und Kritik politisch-ökonomischer Ordnungen. Baden-Baden: Nomos, 339-358.

Scheffer, Thomas (2010): Ethnographie mit System am Beispiel von Englischen Strafverfahren, in: René John/Anna Henkel/Jana Rückert-John (Hrsg.), Die Methodologien des Systems: Wie kommt man zum Fall und wie dahinter. Wiesbaden: VS, 141-160.

Schmidt, Axel (2017): Anpassung an prospektive Zuschauer? Eine multimodal-interaktionsanalytische Perspektive auf Publikums-Konstruktionen in Theaterproben. Zeitschrift für Literaturwissenschaft und Linguistik 47, 455-486.

Schmitt-Beck, Rüdiger (1990): Über die Bedeutung der Massenmedien für die Mobilisierung sozialer Bewegungen. Kölner Zeitschrift für Soziologie und Sozialpsychologie 42, 642-663.

Simmel, Georg (1992) [1908]: Soziologie. Untersuchungen über die Formen der Vergesellschaftung. Frankfurt am Main: Suhrkamp.

Sutter, Tilmann (2014): Folgen gesellschaftlicher Differenzierung: Normativismus, Subjektivismus, Schlichtung „Stuttgart 21“. Zeitschrift für Theoretische Soziologie 3, 295-303.

Starystach, Sebastian (2017): Zur Funktion informeller Kommunikation in Gerichtsverfahren am Beispiel des deutschen Strafverfahrens, in diesem Heft.

Strauss, Anselm (1998): Grundlagen qualitativer Sozialforschung, München: Fink.

Stuckenbrock, Uwe (2013): Das Projekt „Stuttgart 21“ im zeitlichen Überblick, in: Frank Brettschneider/Wolfgang Schuster (Hrsg.), Stuttgart 21. Ein Großprojekt zwischen Protest und Akzeptanz. Wiesbaden: Springer, 15-76.

Tarrow, Sidney (1995): Cycles of Collective Action: Between Movements of Madness and the Repertoire of Contention, in: Mark Traugott (Hrsg.), Repertoires and Cycles of Collective Action. Durham/London: Duke University Press, 89-116.

Tilly, Charles/Tarrow, Sidney (2007): Contentious Politics. Boulder: Paradigm.

Tratschin, Luca (2016): Protest und Selbstbeschreibung. Selbstbezüglichkeit und Umweltverhältnisse sozialer Bewegungen. Bielefeld: transcript.

Tratschin, Luca (2019): Streit vor Publikum. Öffentliche Darstellung von Publikumsgunst als Bezugsproblem sozialer Bewegungen und der Adressaten ihrer Proteste. Zeitschrift für Theoretische Soziologie 8, 2, 194-219.

Vorländer, Hans et al. (2016): PEGIDA. Entwicklung, Zusammensetzung und Deutung einer Empörungsbewegung. Wiesbaden: Springer VS.

\section{Sonstige Quellen}

Bahners, Patrick (2010): Was heißt Legitimation durch Verfahren? Frankfurter Allgemeine Zeitung, 20.10.2010. http://www.faz.net/aktuell/feuilleton/stuttgart-21-was-heisstlegitimation-durch-verfahren-11054317.html (zuletzt aufgerufen am 02.02.2020).

Baur, Tilman (2018): 400. Montagsdemo in Stuttgart. Die Hoffnung auf den S21-Ausstieg lebt. Stuttgarter Zeitung Online, 15.01.2018. https://www.stuttgarter-zeitung.de/inhalt.s-21-in- 
stuttgart-die-hoffnung-auf-den-ausstieg-lebt.fbe593ab-cde6-4792-a10f-e49694ee45c3. html (zuletzt aufgerufen am 10.02.2020).

Das Erste (2018): Einschaltquoten und Marktanteile deutscher Fernsehsender. https://www. daserste.de/programm/quoten.asp (zuletzt aufgerufen am 25.06.2018).

Grzeschik, David (2018): „Tagesschau“ auf Rekordkurs, herbe Verluste für ProSiebens „Newstime“, Quotenmeter. http://www.quotenmeter.de/n/98143/tagesschau-aufrekordkurs-herbe-verluste-fuer-prosiebens-newstime (zuletzt aufgerufen am 02.02.2020).

Heffner, Markus (2012): Montagdemo findet wieder am Bahnhof statt, in: Stuttgarter Zeitung, 26. November. https://www.stuttgarter-zeitung.de/inhalt.stuttgart-21-montagsdemofindet-wieder-am-bahnhof-statt.f9a7f1d7-8455-4da5-a6e3-b4c3a21ba2e0.html (zuletzt aufgerufen am 02.02.2020).

hil/DPAD/dpa (2010): Stuttgart 21. Neue Massendemo gegen Bahnhofsprojekt. Spiegel Online, 09.10.2010. http://www.spiegel.de/politik/deutschland/stuttgart-21-neue-massendemogegen-bahnhofsprojekt-a-722269.html (zuletzt aufgerufen am 02.02.2020).

hip (2010): Stuttgart 21 Schlichtung. TV-Sender erzielen Spitzen-Quote, in: Stuttgarter Nachrichten, 25. Oktober. https://www.stuttgarter-nachrichten.de/inhalt.stuttgart-21schlichtung-tv-sender-erzielen-spitzen-quote.b61dd8e9-7516-45ea-bea8-c73e8323ac54. html (zuletzt aufgerufen am 02.02.2020).

Schlichtungsverfahren zu Stuttgart 21 (2010 a): Ohne Titel, Stenografisches Protokoll, Stuttgart 22. Oktober 2010. Protokollführer: Henrik Dransmann und Ulrike Schmick. http://www.schlichtung-s21.de/fileadmin/schlichtungs21/Redaktion/pdf/101022/ 22_10_2010_Wortprotokoll_Schlichtungsgespraech_S21.pdf (zuletzt aufgerufen am 02.02.2020).

Schlichtungsverfahren zu Stuttgart 21 (2010 b): Leistungsfähigkeit des Bahnknotens Stuttgart. Stenografisches Protokoll. Stuttgart, 29. Oktober 2010. Protokollführer: Henrik Dransmann und Ulrike Schmick. http://www.schlichtung-s21.de/fileadmin/schlichtungs21/Redaktion/ pdf/101029/2010-10-29-1_A_Wortprotokoll_2._Schlichtungsgespraech.pdf (zuletzt aufgerufen am 02.02.2020).

Schlichtungsverfahren zu Stuttgart 21 (2010 c): Neubaustrecke Wendlingen-Ulm. Kopfbahnhof 21. Stenografisches Protokoll. Stuttgart, 4. November 2010. Protokollführer: keine Angaben. http://www.schlichtung-s21.de/fileadmin/schlichtungs21/Redaktion/ pdf/101104/2010-11-04_Wortprotokoll.pdf (zuletzt aufgerufen am 02.02.2020).

Schlichtungsverfahren zu Stuttgart 21 (2010 d): Kopfbahnhof 21. Auswirkungen von Stuttgart 21 auf den Regional- und Nahverkehr. Besprechung offener Fragen aus den drei vorausgegangenen Schlichtungsgesprächen. Stenographisches Protokoll. Stuttgart, 12. November 2010. Protokollführer: keine Angaben. http://www.schlichtung-s21.de/fileadmin/ schlichtungs21/Redaktion/pdf/101112/2010-11-12_Wortprotokoll.pdf (zuletzt aufgerufen am 02.02.2020).

Schlichtungsverfahren zu Stuttgart 21 (2010 e): Geologie. Sicherheit und Baulauf. Stenographisches Protokoll. Stuttgart, 20. November 2010. Protokollführer: Stefan Ernst und Christoph Filla. http://www.schlichtung-s21.de/fileadmin/schlichtungs21/Redaktion/ pdf/101120/2010-11-20_Wortprotokoll.pdf (zuletzt aufgerufen am 02.02.2020).

Schlichtungsverfahren zu Stuttgart 21 (2010 f): Kosten und Wirtschaftlichkeitsrechung. Stenografisches Protokoll. Stuttgart, 26. November 2010. Protokollführer: keine Angaben. http://www.schlichtung-s21.de/fileadmin/schlichtungs21/Redaktion/ pdf/101126/Wortprotokoll_2010-11-26_korrigiert.pdf (zuletzt aufgerufen am 02.02.2020). 
Schlichtungsverfahren zu Stuttgart 21 (2010 g): Abschlussplädoyers der Projektbefürworter und der Projektkritiker. Empfehlungen des Schlichters Dr. Heiner Geissler. Stenografisches Protokoll. Stuttgart, 30. November 2010. Protokollführer: keine Angaben. http://www. schlichtung-s21.de/fileadmin/schlichtungs21/Redaktion/pdf/101130/2010-11-30\%20 Wortprotokoll.pdf (zuletzt aufgerufen am 02.02.2020).

VSZV (Verband Südwestdeutscher Zeitungsverleger e.V.) (2010): Jahresbericht 2010. https:// vszv.de/wp-content/uploads/2017/03/VSZV-Jahresbericht-2010.pdf (zuletzt aufgerufen am 02.02.2020).

\title{
Autoreninformation:
}

\author{
Dr. Luca Tratschin \\ Universität Zürich, Center for Higher Education and Science Studies (CHESS), Andreasstrasse \\ 15, 8050 Zürich, Schweiz, email: luca.tratschin@chess.uzh.ch
}

Luca Tratschin (Dr. phil.) ist wissenschaftlicher Projektleiter am Kompetenzzentrum für Hochschul- und Wissenschaftsforschung der Universität Zürich und Lehrbeauftragter am Soziologischen Seminar der Universität Luzern. Seine Forschungsinteressen liegen in der politischen Soziologie, der Wissen(schaft)ssoziologie sowie der Organisationssoziologie. Wichtige Publikationen: (2019) Streit vor Publikum: Öffentliche Darstellung von Publikumsgunst als gemeinsames Bezugsproblem sozialer Bewegungen und der Adressaten ihrer Proteste, in: Zeitschrift für Theoretische Soziologie 8, 2. (2017) Dissent Without Disaster. Konditionierte Konflikte und öffentlich appräsentierte Dritte, in: Henrik Gummert et al. (Hrsg.), Medien und Kulturen des Konflikts: Pluralität und Dynamik von Gewalt, Bildung und Generation. Wiesbaden: VS (mit Adrian Itschert). (2016) Protest und Selbstbeschreibung: Selbstbezüglichkeit und Umweltverhältnisse sozialer Bewegungen. Bielefeld: transcript. 